\title{
TOPOGRAFIA, DEMOGRAFIA E (DIS)UNITÀ POLITICA DELLA CITTÀ: CONSIDERAZIONI SU ARIST. POL. 5.3.1303B 7-12*
}

\author{
LAURA LODDO $(*)$
}

Nota presentata dal m.e. Cinzia Bearzot

(Adunanza del 3 giugno 2021)

SuNTO. - Lo scopo di questo articolo è offrire una analisi complessiva della riflessione di Aristotele sulla stasis dia tous topous, cioè del caso in cui la conflittualità civica si spiega a partire da una particolare conformazione del territorio che impedisce l'unità politica della polis. A questo scopo, si analizzano innanzitutto gli esempi storici impiegati nell'esempio e si illustrano i dati disponibili sugli episodi di stasis che coinvolsero le città protagoniste dell'esempio aristotelico e si verifica la congruenza fra ogni episodio di stasis concernente queste città e il passo di Aristotele sulla stasis dia tous topous. In seguito, ci si concentra sull'esempio concernente Atene. Si sostiene che il caso ateniese è esemplificativo della disomogeneità socio-politica di una polis che si traduce nella corrispondenza fra residenza e orientamento politico. Non c'è ragione, dunque, di ammettere nell'esempio ateniese un riferimento preciso a una guerra civile reale; piuttosto, esso deve essere messo in connessione con alcuni capisaldi del pensiero di Aristotele: il collegamento fra la flotta e la democrazia e la coincidenza fra lo stile di vita individuale e l'appartenenza ad un determinato gruppo socio-politico.

$* * *$

ABSTRACT. - The aim of this article is to offer a comprehensive analysis of Aristotle's reflection on the stasis dia tous topous, i.e. the instance in which civic conflict is explained as a consequence of a particular layout of the territory that impedes the political unity of the polis. We first analyse the available data on the episodes of stasis that involve the cities in Aristotle's example, in order to estimate the degree of congruence between each episode of stasis occurred in these cities and Aristotle's passage on the stasis dia tous

(") Università di Cagliari, Dipartimento di Lettere, Lingue e Beni Culturali, Italy. E-mail: laura.loddo@unica.it 
topous. Second, we focus on the example concerning Athens, which, it is argued, illustrates the socio-political heterogeneity of a polis, which culminates in the correspondence between residence and political orientation. Hence there is no reason to see in the example of Athens a precise reference to an actual civil war but rather to some cornerstones of Aristotle's thought, i.e. the connection between the fleet and democracy, and the coincidence between individual lifestyle and membership in a certain socio-political group.

\section{INTRODUZIONE}

Nell'Atene classica operazioni su larga scala di epurazione dei cittadini furono realizzate sotto regimi antidemocratici e furono per lo più condotte mediante la violenza fisica e la politica dell'intimidazione². L'esclusione dal corpo civico comportava, fra le altre cose, la separazione fisica degli esclusi dalla sede del potere. Ciò non significava, tuttavia, che gli epurati fossero allontanati sempre e in ogni caso dalla città. Gli esclusi potevano essere ricollocati mediante un trasferimento forzato all'interno del paese, piuttosto che divenire oggetto di un'espulsione formale dall'intero territorio ${ }^{3}$. Si tratta di una questione ben nota e delicata che anche i teorici antichi hanno affrontato. Aristotele ha preso in considerazione questa situazione concreta quando ha tentato di definire cosa fosse una polis e come e per quali fattori essa fosse sottoposta al cambiamento. Infatti, nel chiedersi in quali circostanze un insieme di uomini che abitavano lo stesso luogo fosse da considerarsi una comu-

1 Mi sia consentito esprimere un sentito ringraziamento alla prof.ssa Cinzia Bearzot per l'invito rivoltomi a presentare gli esiti di questa ricerca presso l'Istituto Lombardo. Desidero ringraziare inoltre la dott.ssa Elena Langella per aver generosamente condiviso con me alcune importanti osservazioni sul passo di Aristotele che è oggetto di questo saggio.

2 Per una visione di insieme dei casi ateniesi si veda Bearzot 1994, che segnala come sia possibile seguire questo schema nelle fonti soprattutto per i casi del 404/3 e del 322/18 a.C. e, più recentemente, Costanzi 2020, 17-19, che include anche i casi di emigrazione forzata e deportazione occorsi nelle città della Sicilia, specie in connessione con la politica dei Dinomenidi. In generale per il ricorso alla deportazione si veda Garland 2014, 79-97. Per gli esili comminati sotto i Trenta si rimanda a Seibert 1979, 90-91, 468 nn. 718-727; Forsdyke 2005, 195-204; Garland 2014, 94-96; Bearzot 2020; per la deportazione di massa sotto Antipatro vedi Seibert 1979, 163-165, 512-513 nn. 1285-1296 e ora Poddighe 2020 con ampia discussione della bibliografia precedente.

3 Così effettivamente avvenne nel 404/3 ad Atene. Cf. ultra $\$ 2.3$. 
nità di cittadini, il filosofo ha esaminato diverse ragioni che possono mettere in discussione la natura di una polis: i cambiamenti di regime ${ }^{4}$, le alterazioni dell'equilibrio demografico dovute al ciclo di nascite e morti dei cittadini ${ }^{5}$, il trasferimento di parte della popolazione in un luogo diverso da quello originario ${ }^{6}$. Quest'ultimo riferimento dovrebbe essere collegato a ciò che Aristotele considerava una delle condizioni fondamentali per parlare di una polis, cioè l'appartenenza allo stesso territorio e la conseguente condivisione dello spazio civico ${ }^{7}$. Naturalmente, i cittadini di una stessa polis devono avere molto altro in comune: devono vivere una vita in comune, prevedere riunioni periodiche, condividere scopi e progettualità perché la città è un'associazione di persone simili che si pongono come fine ultimo la felicità ${ }^{8}$. Tuttavia, ci sono molti passi della Politica in cui il filosofo minimizza l'importanza dell'unità territoriale per valutare l'esistenza di una polis'. Esemplare è la sua osservazione sull'eventuale unione tra Megara e Corinto, due città che si trovavano a soli 30 chilometri di distanza l'una dall'altra:

"Infatti, se si dovesse effettivamente riunire i siti di due città in uno solo, in modo che le mura di Megara e quelle di Corinto fossero contigue, anche così non sarebbero una sola città" ${ }^{10}$.

Il ricorso da parte di Aristotele a questo exemplum fictum serve a dimostrare che l'unione artificiale di due territori non è condizione sufficiente a formare una nuova città. In effetti, la posizione di Aristotele trova un riscontro chiaro nell'esperienza concreta ${ }^{11}$, giacché esperimenti simili furono tentati nell'antichità senza molto successo. Durante la guerra di Corinto la città di Argo impose a Corinto un sinecismo anomalo, proba-

\footnotetext{
4 Arist. Pol. III, 1.1274b 34-37; III, 2.1276a 8-16.

5 Arist. Pol. III, 3.1276a 34-1276b 1.

6 Arist. Pol. III, 3.1276a 17-19.

7 Arist. Pol. II, 1.1260b 40-41; III, 9.1280b 14-36. Cf. Schütrumpf-Gehrke 1996, 463.

8 Arist. Pol. III, 9.1280b 24-40; III, 13.1284a 2; VII, 8.1328a 25 ss., 36-40.

9 Arist. Pol. II, 5.1263b 35-36; III, 4.1276b 29; III, 8.1280b 13-15; VII, 4.1326a 7-8. Cf. also Eth. Nic. IX, 9.1170b 11.

10 Arist. Pol. III, 8.1280b 13-15.

11 Ciò vale allo stesso modo per la narrazione di episodi di stasis da parte di Tucidide. Cf. Zatta 2010.
} 
bilmente una convenzione di isopoliteia ${ }^{12}$, per effetto del quale gli abitanti di Corinto, almeno nella prospettiva, innegabilmente orientata, dei Corinzi filo-spartani, furono ridotti alla condizione di meteci nel loro stesso territorio ${ }^{13}$. Non è improbabile che Aristotele abbia in mente esempi come questo quando parla dell'unione tra Megara e Corinto. La minore enfasi che Aristotele pone sull'unità territoriale della polis si può far derivare ragionevolmente dal presupposto che la residenza in uno stesso luogo, agli occhi del filosofo, non rappresenta da solo un criterio adeguato per definire l'appartenenza alla comunità poleica. Ciononostante, nella riflessione aristotelica la topografia di un luogo è un aspetto chiave per comprendere l'evoluzione delle relazioni tra i cittadini. In generale, era sua convinzione che la conformazione del territorio potesse influenzare la vita politica dei cittadini, le interazioni quotidiane e la dialettica politica e favorire l'emergere di contrapposizioni. Più precisamente, nel libro 5 della Politica Aristotele sostiene che in alcuni casi l'esplosione del conflitto civico risulta causato dalle caratteristiche fisiche del territorio, poiché il luogo in cui sorge la polis può ostacolare l'unità politica, promuovere divisioni, esacerbare le differenze.

Talora le città cadono in conflitto civile anche a causa della posizione geografica, quando il luogo non sia idoneo per natura all'esistenza di una sola città: a Clazomene, per esempio, gli abitanti di Chito lottano con quelli dell'isola, e così gli abitanti di Colofone con quelli di Notio. Anche ad Atene gli abitanti non hanno le stesse disposizioni, ma quelli del Pireo sono più democratici di quelli della città. Nello stesso modo in cui,

12 Oltre ai problemi di datazione, su cui fa il punto Sordi 2006, 299-300 e n. 3 , molta attenzione è stata rivolta alla natura dell'unione. Taluni hanno parlato di sinecismo (così Moggi 1976, 242-251, nr. 39); altri di isopoliteia (Griffith 1950, 247-248; Whitby 1984, 298 n. 13; Gehrke 1985, 85 n. 22; Bearzot 2004, 31-36, Sordi 2006, $304-$ 308; Bettalli 2012, 171 e n. 27); altri ancora di sympoliteia (Buckler 2003, 105-106, secondo cui "they began to incorporate the polis of Corinth into that of Argos in what was ostensibly called unification, either isopoliteia or perhaps better sympoliteia"). Diversa la posizione di Fornis 2006, che nega la natura di esperimento politico dell'unione e riconduce l'episodio al prolungarsi della stasis a Corinto, in cui la fazione minoritaria, di orientamento oligarchico e filo-argiva, per imporsi sulla fazione filo-laconica, anch'essa oligarchica, avrebbe avuto necessità di chiamare in aiuto Argo e la sua forza militare. $\mathrm{Ma}$ è difficile ammettere che la componente filo-argiva tenesse una posizione antidemocratica.

13 Xen. Hell. IV, 4.1-7; IV, 5.3; IV, 8.34; V, 1.34; Andoc. 3.27; Dem. 20.51-53; Diod. Sic. XIV, 86.1; XIV, 92.1; XV, 40.3. 
durante le guerre, l'attraversamento dei fossati, sebbene essi siano davvero piccoli, turba l'ordine della falange, così è naturale che ogni differenza produca un contrasto. Dunque, l'opposizione più grande è forse quella fra virtù e vizio, poi fra ricchezza e povertà e così via una più di un'altra e una di queste è la differenza che è stata appena detta ${ }^{14}$.

Sono tre gli esempi addotti da Aristotele per corroborare il suo assunto: l'opposizione a Clazomene tra coloro che vivono sul continente

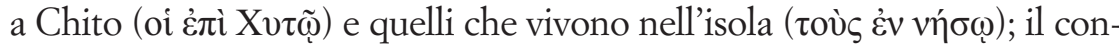
flitto tra i Colofoni residenti in città e quelli emigrati nella zona di Nozio; la divisione all'interno di Atene tra coloro che abitano al Pireo, considerati più democratici, e quelli che popolano il centro urbano, l'asty. A ben guardare, questi esempi non sono esattamente sovrapponibili, ma presentano alcune caratteristiche comuni. L'esistenza di una chiara polarizzazione tra gruppi politici opposti, in politica interna come in politica estera, è la vera ragione dello scoppio delle lotte civili, ma questo stato di cose può essere talvolta implementato da fattori potenzialmente divisivi, quali la conformazione geografica del territorio e la distribuzione della popolazione nello spazio della città. Allo stesso modo, in Pol. VII, 11.1330b 17-21 Aristotele, trattando dei luoghi fortificati, collega luoghi specifici della città con particolari regimi politici, per cui l'acropoli si confà essenzialmente a una oligarchia o a una monarchia, la pianura alle democrazie, mentre all'aristocrazia si addicono piuttosto luoghi fortificati. ${ }^{15}$ Non si tratta, però, di una visione deterministica applicata all'interpretazione della stasi ${ }^{16}$, secondo la quale la conformazione territoriale di una città definisce la sua tendenza al conflitto, come potrebbe sembrare ad una

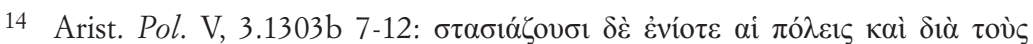

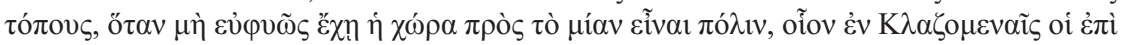

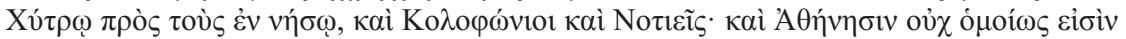

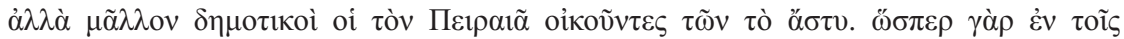

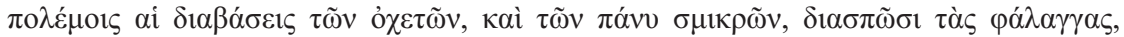

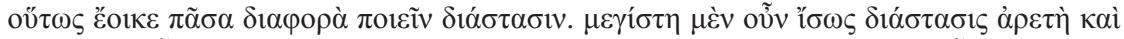

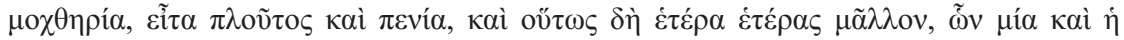

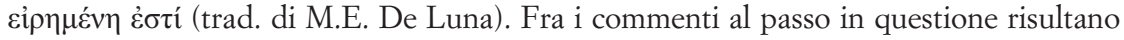
utili quelli di Aubonnet 1973, 162-164; Schütrumpf-Gehrke 1996, 463-465; De Luna 2016, 282, 315-322.

15 Per un'analisi cfr. ora Carsana-Zizza 2019, 172.

16 Ragone 2005, 11 n. 18, $20 \mathrm{nn}$. 71-72, a proposito dell'analisi di Aristotele circa la contrapposizione fra Colofone e Nozio, parla di "opinabile determinismo geografico". 
prima lettura, ma dell'enunciazione di un principio elaborato su base empirica, cioè l'osservazione e lo studio della stasis nelle poleis così conformate. Questo principio si rivela decisivo nel prolungare la stasis quando la città manca di unità politica, avversando il raggiungimento della concordia e lasciando prevalere le differenze.

La stasis dia tous topous è stata oggetto di una certa attenzione da parte della critica in rapporto sia alla teoria aristotelica generale sulla stasi $^{17}{ }^{17}$ sia all'efficacia della costruzione teorica per spiegare singole realtà storiche. È quest'ultimo versante ad interessare questo articolo. I principali argomenti di discussione in questo ambito sono due. Il primo riguarda l'affidabilità dell'interpretazione di Aristotele sul presunto contrasto tra le città il cui territorio si sviluppa originariamente nell'entroterra e le loro aree portuali, quando queste assumono un'importanza tale da oscurare la centralità del centro cittadino primario. Mentre alcuni studiosi hanno dato credito ad Aristotele per quanto riguarda l'opposizione tra gli abitanti del Pireo e quelli dell'asty ${ }^{18}$, altri hanno sottolineato che non si hanno prove di una opposizione in termini socio-politici fra diverse zone della città. Il Pireo, come in generale le aree portuali nella riflessione greca, rappresenterebbe per così dire una superficie di proiezione di pregiudizi morali diffusi ${ }^{19}$. Allo stesso modo è stata messa in dubbio la bipolarizzazione fra Colofone e il porto di Nozio che Aristotele riconduce alla conformazione disomogenea del territorio; piuttosto, si è detto, il carattere ricorrente delle staseis che coinvolsero Colofone e Nozio rifletterebbe "difficoltà politiche oggettivamente in atto in età classica" ${ }^{20}$. Il significato generale del passo costituisce un secondo argomento di discussione. Questi i termini: quando Aristotele ricorre agli esempi di Colofone, Clazomene e Atene, si riferisce a casi noti di stasis, soffermandosi su momenti specifici della storia di quelle città in cui la conflittualità latente si tradusse in scontri aperti ${ }^{21}$ ? O piuttosto egli elenca esempi di città la cui storia è caratterizzata da conflitti

17 Contogiorgis 1978, 184-186; Weed 2007, 59-60; Rogan 2018, 101, 107-111; De Luna 2020, 9-11.

18 Amit 1961, 471; Garland 1987.

19 Von Reden 1995; Roy 1998; Schmidt 2014, 640-641.

20 Ragone 2005, 20. Contra De Luna 2020, 13-17.

21 Schütrumpf-Gehrke 1996 e De Luna 2016 e 2020, 12-13, ritengono generici i riferimenti alle staseis di Clazomene e Colofone, ma puntuale il riferimento alla contrapposizione fra Atene e il Pireo. Per Daverio Rocchi 2004, 299 "il riferimento 
esacerbati dalla loro particolare conformazione geografica, dove cioè si nota una tendenza alla polarizzazione che esula dalla sua possibile traduzione in episodi di lotte civili ${ }^{22}$ ? Discernere come Aristotele abbia proceduto è operazione resa più difficile dal fatto che, nel trattare il cambiamento politico che si attua nel contesto della stasis, Aristotele spesso cita un episodio specifico senza fornire un elenco né tantomeno un'analisi degli altri fattori che hanno concorso a determinarlo ${ }^{23}$.

Questo articolo si propone di riconsiderare il resoconto di Aristotele sulla stasis dia tous topous nel suo complesso, con l'obiettivo di mettere a fuoco la funzione degli esempi storici impiegati.

A questo scopo, la prima sezione offre una panoramica dei dati disponibili sugli episodi di stasis che coinvolsero le città protagoniste dell'esempio aristotelico e verifica la congruenza fra ogni episodio di stasis concernente queste città e il passo di Aristotele sulla stasis dia tous topous. La seconda sezione prende in considerazione il caso specifico di Atene: vi si espongono le ragioni che ci impediscono di collegare il passo con un preciso episodio di stasis e si esclude che Aristotele abbia basato la sua affermazione sulla guerra civile che sconvolse Atene nel 404/3 all'epoca dei Trenta; vi si sostiene, inoltre, che nella definizione degli abitanti del Pireo come più $\delta \eta$ ноєкоí si debba leggere non solo l'impegno a favore della democrazia, ma una precisa correlazione tra appartenenza politi$\mathrm{ca}$, posizione sociale e status occupazionale. Questo è qualcosa di più di una semplice lettura socio-politica. Accettando il principio che il regime politico e il modo di vivere si influenzano a vicenda, principio che è ben attestato negli scritti politici aristotelici, il filosofo sembra riferirsi a una concezione diffusa negli scrittori contemporanei, conferendole dignità teorica attraverso la sua teoria della stasis. Aristotele, lungi dall'adottare un approccio deterministico alla interpretazione della stasis dia tous topous, ritiene in definitiva che la divisione territoriale, elemento di per sé non politico che tende a diventare tale in congiunzione con fattori propriamente politici ${ }^{24}$, favorisce l'emergere di una identità specifica e agevo-

aristotelico agli abitanti del Pireo più demotikoi di quelli dell'asty non esclude una lettura selettiva, politicamente circostanziata in relazione ai fatti del 403, secondo l'esegesi tradizionale, ma nell'equilibrio del passo del libro V della Politica sembra acquistare una risonanza più modulata".

22 Garland 1987; Von Reden 1995; Roy 1998.

23 Lintott $2013^{2}, 241,248$.

24 Phillips Simpson 1998, 377. 
la la legittimazione politica di gruppi dissimili; essa, tuttavia, non sfocia nella frammentazione e nella contrapposizione politica in tutte le società, bensì solo in quelle in cui $\mathrm{i}$ gruppi sociali non hanno imparato a cooperare e a ricomporre il conflitto. Altri fattori, in effetti, paiono realmente decisivi nell'avviare la stasis.

\section{Aristotele E LA CONFORMAZIONE DEI LUOGHi COME CAUSA DELLA STASIS}

La teoria aristotelica sulla stasi $^{25}$ ha tralasciato ogni accenno ad una causa formale ${ }^{26}$. Nell'ambito della sua indagine sulle fonti del cambiamento politico, che non è solo teorica, ma guarda alle implicazioni pratiche della conflittualità civica ${ }^{27}$, Aristotele sembra essere molto più

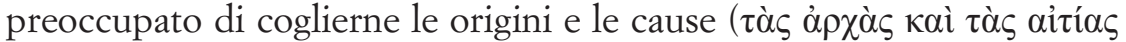
$\alpha \hat{\tau} \tau \tilde{\omega})^{28}$. Ciascuna delle tre cause individuate - lo stato d'animo degli individui coinvolti nella stasis; gli obiettivi che spingono le parti al conflitto; le cause occasionali - viene discussa più in dettaglio ${ }^{29}$. In primo luogo, Aristotele rivolge l'attenzione alla relazione che intercorre fra la stasis e la condizione di coloro che avviano una rivolta, individuandone la causa principale in una diversa concezione della giustizia: alcuni sembrano aspirare all'uguaglianza nella convinzione di avere meno degli altri, mentre altri mirano alla disuguaglianza o ai privilegi quando, nella convinzione di meritare di più, pensano di avere meno degli altri ${ }^{30}$. In secondo luogo, egli indica sette (e forse più) cause di cambiamento che sono parzialmente legate ai fattori già descritti: due di esse riguardano il sentimento di ingiustizia derivante dal presupposto che l'altra parte

25 Va oltre gli obiettivi di questo saggio offrire una trattazione sulla stasis in Aristotele, soggetto peraltro molto indagato anche in tempi recenti. Di seguito una selezione di contributi sul tema che ritengo significativi: Contogiorgis 1978; Kalimitzis 2000; Weed 2007; Lintott 2013², 238-250; Pellegrin 2017, 358-377; Rogan 2018; Brill 2020, 187-190; Cairns, Canevaro, Mantzouranis 2021.

26 Le ragioni di questa mancanza possono essere individuate nell'impossibilità di offrire una definizione di stasis priva di ambiguità. Cf. Kalimitzis 2000, 149-156.

27 Lintott 2018, 11.

28 Arist. Pol. V, 2.1302a 16-18.

29 Arist. Pol. V, 2.1302a 22-1302b 5.

30 Arist. Pol. V, 2.1302b 22-31. Cfr. V, 1.1301a 33-b 4, 1301b 35-39. 
abbia ricevuto qualche guadagno o onore che non merita ${ }^{31}$. Oltre a questi aspetti egli elenca l'arroganza, la paura, la superiorità, il disprezzo e la crescita sproporzionata come cause che innescano la stasis ${ }^{32}$. Altre cause sono menzionate in connessione con il cambiamento della forma di governo (gli intrighi elettorali, la negligenza, la disattenzione, la dissomiglianza degli elementi di una città) ${ }^{33}$, ma solo la dissomiglianza può provocare la faziosità che evolve nella stasis.

È convinzione di Aristotele che ci siano cause oggettive in grado di scatenare disordini civili. Tra le cause oggettive egli elenca sia l'eterogeneità razziale ${ }^{34}$ sia le cause endogene ${ }^{35}$. La prima è intesa come il risultato di abitudini e concezioni di vita differenti, instillate permanentemente negli individui nel corso del tempo a causa delle diverse condizioni sociali, politiche e climatiche in cui hanno vissuto e delle convinzioni morali che hanno acquisito ${ }^{36}$. La diversità etnica è un fattore latente di conflitto fintantoché i diversi elementi che compongono la polis non apprendano a convivere in armonia. L'importanza e l'incidenza che Aristotele attribuisce alla differenza etnica nella genesi e nella proliferazione del conflitto civico sono evidenti dal gran numero di eventi storici e dall'abbondanza di esempi concreti ai quali il filosofo ricorre come paradeigmata ${ }^{37}$.

$\grave{\mathrm{E}}$ in questo quadro che Aristotele introduce il suo trattamento

31 A una prima lettura la lista delle cause del cambiamento non sembra corrispondere alle sette cause che Aristotele individua. Sono sette solo se si considera che il profitto e l'onore sono due cause che operano in due modi differenti.

32 Arist. Pol. V, 2.1302b 2-3. Ciascuna di queste cause è trattata in modo più approfondito in Pol. V, 3.1302b 5-1303a 13, benché la trattazione della stasis $\delta \mathrm{t}$ '

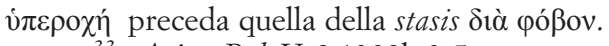

33 Arist. Pol. V, 2.1302b 3-5.

34 Arist. Pol. V, 3.1303a 25-1303b 7.

35 Arist. Pol. V, 3.1303b 7-17.

36 Arist. Pol. VII, 7.1327b 20-36.

37 Sono menzionati 8 esempi: l'espulsione dei Trezeni da parte degli Achei da Sibari alla cui fondazione entrambe le popolazioni avevano contribuito; il conflitto a Turi tra i Sibariti e i loro co-fondatori; l'espulsione dei coloni di seconda generazione (epoikoi) a Bisanzio; l'espulsione da parte degli Antissei degli esuli chii che si erano rifugiati ad Antissa; l'espulsione degli Zanclei da parte dei coloni sami; lo scoppio della stasis ad Apollonia dopo l'ammissione nella città di nuovi coloni; la stasis a Siracusa dopo le concessioni di cittadinanza a mercenari e stranieri; l'espulsione degli Anfipolitani dal loro territorio da parte dei Calcidesi cui gli Anfipolitani avevano concesso asilo. 
della stasis dia tous topous. È stato sottolineato che il conflitto che si origina per la particolare conformazione territoriale della polis è il risultato dell'opposizione tra aree geografiche diverse: la presenza di alture che fungono da elementi separatori può determinare l'opposizione fra pianura e montagna, la vicinanza al mare di una parte del territorio può provocare una tensione fra zona costiera e zona interna o, nel caso di poleis isolane il cui territorio si estende anche sul continente, l'opposizione fra zone continentali e aree insulari ${ }^{38}$. Non sembra, tuttavia, che gli esempi impiegati da Aristotele si spieghino a partire da queste contrapposizioni. È più corretto affermare che questa tensione accomuna solo i primi due esempi (Clazomene/Chito e Colofone/Nozio), mentre il caso di Atene serve ad illustrare un altro assunto. Il riferimento alla divisione fra gli abitanti del centro urbano e quelli del Pireo si lega piuttosto all'osservazione successiva, anticipata dalla metafora della falange, che ogni differenza nella città produce potenzialmente un contrasto. Il caso ateniese è esemplificativo della disomogeneità socio-politica di una polis che si traduce nella corrispondenza fra residenza e orientamento politico. Non c'è ragione, dunque, di ammettere un riferimento preciso a una guerra civile reale - fattori linguistici e considerazioni di ordine storico concorrono a dimostrarlo; piuttosto, il passo deve essere messo in connessione con alcuni capisaldi del pensiero di Aristotele: il collegamento fra la flotta e la democrazia e la coincidenza fra lo stile di vita individuale e l'appartenenza ad un determinato gruppo socio-politico.

\subsection{Clazomene/Chito}

Il primo caso riguarda le relazioni tra l'isola di Clazomene e Chito, un insediamento fortificato sulla sua perea, nella Ionia ${ }^{39}$, che secondo Strabone costituiva l'insediamento originario dei Clazomeni ${ }^{40}$. L'occupazione della perea da parte dei Clazomeni è spesso collegata nelle fonti all'attività degli esuli politici e a diversi episodi di disordini civili. Sappiamo dell'esistenza di tre insediamenti di Clazomene nella

38 Contogiorgis 1978, 187.

39 GHI 18, 11. 9-10. Su Chito vedi Ephorus, BNJ 70 F 78 = Steph. Byz. s.v.

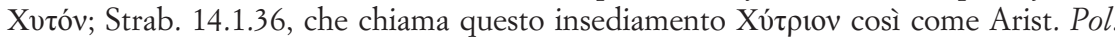
V, 3.1303b 7-10.

40 Strab. XIV, 1.36. 
perea: Policna, Dafnunte e Chito. Mentre l'occupazione di Policna fu di breve durata ${ }^{41}$, gli insediamenti di Dafnunte e Chito ebbero maggiore fortuna. Dopo l'abbandono di Policna, Dafnunte divenne una roccaforte dei Clazomeni filo-spartani. Lo si deduce dal fatto che durante l'ultima fase della guerra del Peloponneso il navarca spartano Astioco diede l'ordine ai filo-spartani di trasferirsi a Dafnunte, segno di un piano spartano per raccogliere gli esuli che simpatizzavano per Sparta nella perea e per sfruttarla come base per le incursioni sull'isola ${ }^{42}$. Il progetto di Astioco fallì, ma la stasis a Clazomene continuò per alcuni anni, almeno fino al 407 a.C., quando, su proposta di Alcibiade, l'assemblea ateniese ratificò gli accordi con quanti si trovavano a Dafnunte. Un decreto riguardante gli accordi tra Atene e Clazomene attesta la distensione dei rapporti tra gli esuli di Dafnunte e i Clazomeni dell'isola in seguito al loro riavvicinamento agli Ateniesi ${ }^{43}$. E probabile, comunque, che una parte degli esuli sia rimasta a Dafnunte. Diodoro (13.71.1) attesta la presenza di esuli clazomeni che piagavano i Clazomeni dell'isola con continue incursioni.

Anche il sito di Chito è legato a una stasis, della cui esistenza si apprende da un documento epigrafico datato al 387/6 a.C. ${ }^{44} \mathrm{Il}$ documento, un decreto onorifico ateniese in cui il popolo dei Clazomeni è elogiato per lo zelo e la fedeltà nei confronti di Atene, prevede una clausola interessante per il nostro discorso. Si tratta della richiesta di non interferenza nei rapporti con gli esuli di Chito (l. 5), avanzata con buona sicurezza dai Clazomeni dell'isola: essi, distinguendosi dagli esuli che occupano Chito, cercano di negoziare i termini dell'egemonia ateniese sull'isola e, nel contempo, di presentarsi al popolo degli Ateniesi come il governo legittimo a Clazomene. L'isola, come comunemente si

41 Nel 412 a.C. i Clazomeni defezionarono dalla Lega Delio-attica ma, temendo la reazione ateniese, lasciarono l'isola e si trasferirono sulla terraferma, fortificando il sito di Policna (Thuc. VIII, 14.1-3). Una volta che gli Ateniesi ripresero il controllo dell'isola, costrinsero i Clazomeni ad abbandonare Policna e a tornare sull'isola, ad eccezione dei capi della rivolta che si stabilirono a Dafnunte (Thuc. VIII, 23.5-6). Le fonti non permettono di chiarire se il loro mancato ritorno sull'isola sia dovuto a una scelta degli interessati, in aperto disaccordo con il resto della popolazione, o se si tratti di un'imposizione ateniese. Cfr. Loddo 2019, 215-216.
42 Thuc. VIII, 31.1-2.
43 IG I3 $119.5=$ GHI 186.
44 GHI 18. 
ammette, era stata recuperata da Atene al tempo del viaggio di Trasibulo nell'Ellesponto, anche se il dato si ricostruisce solo dall'interpretazione dell'iscrizione e non ha riscontro nella tradizione letteraria. Ho discusso altrove la questione del presunto cambiamento costituzionale che avrebbe riguardato Clazomene all'epoca del recupero dell'isola da parte di Trasibulo, argomentando contro l'esistenza di un rivolgimento costituzionale in senso democratico ${ }^{45}$. Piuttosto, pare preferibile interpretare la stasis che coinvolse i Clazomeni come un conflitto derivante dalla contrapposizione fra i filo-ateniesi dell'isola e i filo-persiani del continente: mentre i Clazomeni dell'isola si ponevano sotto la prostasia di Atene, gli esuli di Chito aspiravano a rientrare nell'orbita persiana, facendo leva sulla vocazione continentale di Clazomene e sulla sua storia passata.

In senso stretto la contrapposizione fra quelli dell'isola e gli esuli di Chito non fu un conflitto derivante dalla conformazione geografica del territorio, poiché la fazione espulsa occupò questo insediamento nella terraferma dopo la sua sconfitta politica. L'occupazione della perea, pertanto, non fu la ragione del conflitto, piuttosto una delle sue principali conseguenze. Questa osservazione potrebbe corroborare la tesi di quanti sostengono che, citando il caso di Clazomene-Chito, Aristotele non abbia voluto riferirsi a un episodio specifico del conflitto tra i due insediamenti, ma abbia voluto sottolineare l'esistenza di un conflitto latente che la conformazione geografica di questi insediamenti può aver favorito. Più precisamente, la disponibilità per i fuoriusciti di un territorio vicino in cui installarsi e da cui proseguire la lotta ha contribuito a dilatare il conflitto anche oltre i confini della città. Seppure Aristotele abbia avuto in mente un episodio specifico della storia di Clazomene, cosa che non può essere esclusa in termini assoluti, ciò che importa rilevare è che il riferimento alla peculiare condizione di Clazomene ha lo scopo di illustrare come la conformazione del territorio possa avallare lo scoppio della stasis, favorire la frattura tra le fazioni e stabilizzare tale frattura attraverso la possibilità data alle parti di trasferirsi in luoghi specifici nel territorio della città ${ }^{46}$. È degno di nota che la relazione Clazomene-Chito sia stata accostata a quella che Mitilene intratteneva con le Aktian poleis e al rapporto fra polis primaria e polis

\footnotetext{
45 Loddo 2019, 221-227.

46 De Luna 2016, 315-316.
} 
dipendente ${ }^{47}$ : le espulsioni occorse in questi territori a seguito della stasis equivalgono a esilii (o auto-esilii) interni.

\subsection{Colofone/Nozio}

Il caso di Colofone-Nozio rientra nello schema appena illustrato. Il passo di Aristotele, la cui analisi è oggetto di questo intervento, consente di affermare che il filosofo considerava Nozio una parte della polis di Colofone. Nonostante ciò, la critica ha sottolineato che Nozio, il porto di Colofone, si è evoluto nel tempo in una entità separata, in quella forma ibrida che è la "polis dipendente" ${ }^{48}$. In un decreto di età ellenistica per Magnesia sul Meandro, probabilmente da Clazomene, in cui si concede l'asylia ai Magnesi e lo statuto panellenico alla festa di

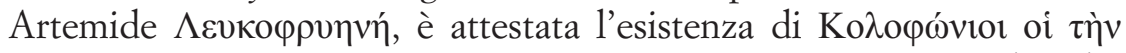

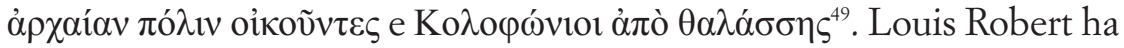
proposto di identificare la prima nella Colofone d'età classica e nella seconda l'insediamento di Nozio ${ }^{50}$. Livio, parlando di Nozio, la definisce oppidum Colophonium e ne calcola la distanza a vetere Colophone in due miglia ${ }^{51}$. I rapporti fra le due città, dunque, vanno mutando nel tempo. Con questa premessa analizziamo possibili contesti della stasis che coinvolse Colofone e Nozio.

Poche sono le notizie su Colofone in età arcaica; tuttavia, i rari riferimenti nelle fonti indicano un ruolo di preminenza del centro colofonio all'interno dello scenario ionico. Non è solo la posizione geografica - la città sorgeva nell'estremità meridionale del promontorio situato fra il territorio di Smirne e quello di Efeso, prospiciente all'isola di $\mathrm{Chio}^{52}$ - ad aver garantito a Colofone un ruolo di primo piano, ma anche la potenza militare che fu alla base della conseguente influenza politica. Il geografo Strabone, a questo proposito, trasmette un motto

47 Hansen-Nielsen 2004, 88-89.

48 Hansen-Nielsen 2004, 92.

49 Iv.Magnesia 53, 11. 75-79 (= I.Erythrai 507).

50 Robert 1962, 62, 272. L'identificazione di Colofone sul mare con Nozio è già in Kern 1900, 44.

51 Liv. XXXVII, 26.5.

52 La descrizione più accurata dello scenario topografico di Colofone e Nozio, con un resoconto delle campagne di scavo che hanno riguardato Colofone, Nozio e Claro, sede di un tempo di Apollo, è quella offerta da Ragone 2005, 11-21. 


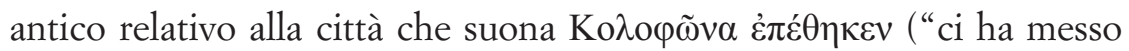
sopra Colofone”), che allude probabilmente al ruolo internazionale assunto da Colofone come risolutrice delle controversie fra le città ${ }^{53}$. Il passo di Strabone è utile a chiarire anche le ragioni all'origine dell'influenza politica di Colofone. Esso allude infatti al possesso di un buon numero di navi e a una forte cavalleria. Ma se l'autorità di Colofone si può comprendere alla luce dei rapporti con le isole vicine e le città continentali e l'allevamento dei cavalli si spiega a partire dalla natura pianeggiante del territorio ${ }^{54}$, la potenza marittima della città è tema spinoso, giacché Nozio non sembra sia stato in grado di concorrere con gli altri porti della Ionia ${ }^{55}$. Ed è forse questa debolezza strutturale ad aver agevolato la conquista della città da parte dei Lidi già all'inizio del VII secolo, quando Colofone, sola fra le città della Ionia, cadde nelle mani di Gige ${ }^{56}$. Il VII secolo sotto la dominazione lidia è un periodo di lotte intestine, in cui il contrasto è prevalentemente all'interno dell'aristocrazia stessa: da un lato i ghene che appoggiano la dominazione dei Lidi, dall'altro alcuni esponenti dell'aristocrazia che, probabilmente appoggiandosi sul sostegno popolare, sostengono il tiranno locale, arroccandosi su posizioni "nazionalistiche". Santo Mazzarino, revocando in dubbio la tesi che attribuiva ai Lidi l'imposizione indifferenziata di tirannidi nella Ionia, ha ricostruito un quadro piuttosto chiaro della politica interna di Colofone e degli altri centri della Ionia, sostenendo che le tirannidi al potere in queste città assunsero una linea politica dichiaratamente anti-lidia ${ }^{57}$. I versi di Senofane sulle áßpoбv́vas

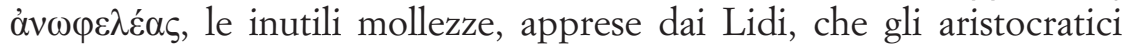
colofoni ostentavano quando si recavano nell'agora ${ }^{58}$, evocano tanto l'e-

53 Strab. XIV, 633. Sul significato del proverbio si rimanda a Fogazza 1974, 29.

54 Parke 1985, 120.

55 Nozio fu il porto meridionale di Colofone forse già in età arcaica, ma non è da escludere che anche la località di Dios Hieron svolgesse una funzione analoga. Cf. Ragone 2005, 19-20 e n. 68. Si deve riconoscere, tuttavia, che Nozio controllava il sito Claros, cf. Parke 1985, 121.

56 Hdt. I, 14.5. Si veda anche Polyaenus, Strat. VII, 2.2 che parla della corruzione e dell'annientamento della cavalleria di Colofone da parte di Aliatte.

57 Mazzarino 1947, 191-203: a Efeso l'opposizione del tiranno Pindaro al re Creso; a Mileto il tiranno Trasibulo guida la resistenza contro Aliatte; a Mitilene i Lidi hanno sostenuto l'aristocrazia locale contro Pittaco.

58 A questo proposito Ruzé 1997, 25-28 interpreta agora come sinonimo di 
sibizione intemperante di una raffinatezza avvertita come non greca, secondo una percezione culturale diffusa, quanto le responsabilità di quel gruppo sociale nell'instaurazione dell'odiosa tirannide ( $\tau$ opavvíns

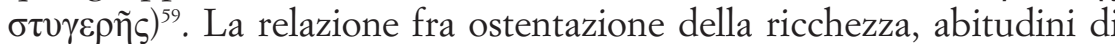
vita e instaurazione della tirannide è resa in termini di causa-effetto in un frammento di Teopompo ${ }^{60}$. È degno di nota che lo storico di Chio illustri il contesto politico in cui si instaura la tirannide, riconducendola a un percorso di progressivo indebolimento del governo in carica, se si può vedere nei Mille dai mantelli di porpora un'allusione al gruppo dirigente colofonio ${ }^{61}$. Teopompo, di cui si conserva anche un frammento su Nozio ${ }^{62}$, non sembra operare una sovra-interpretazione della sua fonte, giacché la correlazione fra stile di vita, caduta del regime vigente e instaurazione della tirannide è presente anche nel poema di Senofane, seppur in forma meno estesa. È questo un possibile contesto della stasis di cui parla Aristotele? Non sembra corretto affermarlo, anche se un passo della Politica è stato spesso messo in relazione con la crisi del VI

Assemblea, ma la sua lettura non sembra condivisibile. Già Teopompo parafrasava l'a-

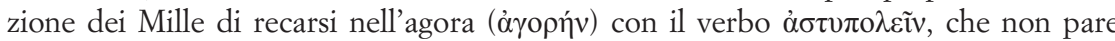
avere carattere tecnico assimilabile al partecipare all'Assemblea. Per quest'ultima interpretazione si veda già Giangiulio 2018, 278 n. 16.

59 Xenophanes, DK 21 B 3 = fr. 3 Reale.

60 Theopomp. FGrHist 115 F 117 = Ath. XII, 31.526c. Cf. anche Phylarchus, FGrHist 81 F 66 = Ath. XII, 31.526a-c.

61 Il punto però è controverso. Mentre in passato si riconosceva in maniera pressoché unanime l'identificazione dei Mille di Colofone con il gruppo dirigente di una oligarchia orientalizzante (Talamo 1973, 353-358; Ragone 1996, 938), interventi recenti hanno messo in discussione questa interpretazione. Duplouy 2013, rifiutando l'approccio istituzionalista allo studio della cittadinanza greca d'età arcaica, considera 1 Mille di Colofone come un'espressione che simbolizza la «totalité symbolique» du corps civique (la citazione, che mantiene inalterato il virgolettato dell'originale, è da p. 164), e spiega l'insistenza delle fonti antiche sulla raffinatezza eccessiva dei Colofoni come una critica non già all'aristocrazia, ma ai costumi di vita tipici del contesto colofonio (e ionico in generale). Anche Giangiulio 2018, 278-280 interpreta i Mille come «the whole community of the citizens and the political body of the polis» (la citazione è da p. 279).

62 Theopomp. FGrHist 115 F 118 = Harp. s.v. Nó 1 ov. Il frammento, concernente la posizione geografica di Nozio, che viene definito un insediamento prospiciente

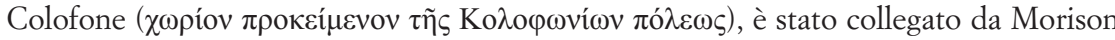
2016 a F 114 (= Ath. XII, 40-41.531a-d): Nozio vi sarebbe menzionata in relazione alla rivolta dei satrapi. A me pare più probabile che Nozio venisse menzionata in connessione con l'excursus su Colofone (F 117), entrambi contenuti nel libro XV dei Philippika (vedi supra n. 60). 
secolo. In Pol. IV, 3.1290a 30-b 21 Aristotele, a cui si fa risalire anche uno scritto sulla politeia di Colofone ${ }^{63}$, inserisce il caso dell'antica

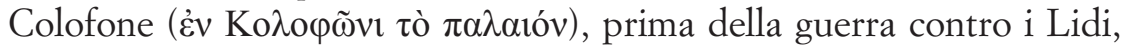
fra gli esempi di quelle forme ibride di regime politico che non possono essere identificate né con la democrazia né con l'oligarchia ${ }^{64}$ : l'antica Colofone non può essere considerata una democrazia perché nelle democrazie a governare sono i molti poveri, ma non si può parlare neanche di oligarchia, se a detenere il potere non sono i pochi ricchi. $\grave{E}$ una determinata combinazione di fattori numerici e socio-economici a definire nella tassonomia aristotelica se una data politeia sia da considerarsi una democrazia o un'oligarchia.

Un altro possibile contesto è rappresentato dalla stasis di V secolo che si consumò durante la guerra del Peloponneso. In verità, nel giro di pochi anni, diversi episodi di conflitto civico scossero Colofone e non è un caso che in tutti questi episodi l'insediamento di Nozio abbia giocato un ruolo importante. La stasis scoppiò la prima volta nel 430, quando la guerra intestina fra i Colofoni si concluse con la conquista della città alta da parte dei Persiani guidati da Itamane, chiamati in aiuto da una delle fazioni in guerra ${ }^{65}$. Una conseguenza importante di questo stato di cose fu l'emigrazione di parte della popolazione a Nozio: si trattò presumibilmente di quegli oppositori che si erano esposti più apertamente e di quella parte che mal tollerava l'occupazione persiana, mentre Colofone rimase con i Persiani almeno fino al 409 a.C., quando gli Ateniesi la riconquistarono ${ }^{66}$. Ma la stasis conobbe una recrudescenza nel 427, al tempo in cui lo stratego ateniese Pachete navigava in Ionia, e coinvolse questa volta l'insediamento di $\mathrm{Nozio}^{67}$. Qui scoppiarono nuovi contrasti. Una delle fazioni in lotta cercò l'appoggio militare per$\operatorname{siano}^{68}$, ricevendo in supporto un manipolo di mercenari arcadi e bar-

63 Si conserva un solo frammento della Kolophonion Politeia (F 514 Rose $=$ Ath. XIV, 10.618c6-619a2 + Poll. IV, 55). Di Colofone Aristotele parlava anche nel F 584 Rose (= Ath. XII, $25.523 \mathrm{~cd}$ ) a proposito della fondazione di Siri da parte dei Colofoni e della diffusione nella colonia della tryphé.

64 Così Giangiulio 2018, 279.

65 Thuc. III, 34.1.

66 Xen. Hell. I, 2.4.

67 Thuc. III, 34.2-3.

68 Tucidide non definisce l'orientamento politico di questa fazione. Gehrke 1985, 80-81 li considera "eine propersische bzw. Athenfeindliche Gruppe, wohl 
bari al soldo di Pissutne ${ }^{69}$; la città di Nozio venne divisa in due zone grazie alla costruzione di un muro fortificato $(\delta 1 \alpha \tau \varepsilon i ́ \chi 1 \sigma \mu \alpha)$, dove, protetti dalla guarnigione persiana, la fazione filo-persiana e i medizzanti fra i Colofoni della città alta detenevano il potere, una volta cacciati i filoateniesi. Fu questa fazione in esilio a chiamare in aiuto gli Ateniesi, la cui risposta fu tempestiva. Lo stratego Pachete conquistò il luogo fortificato, fece una carneficina dei mercenari lì asserragliati, espulse i filopersiani e consegnò la città ai Colofoni che non avevano medizzato. A

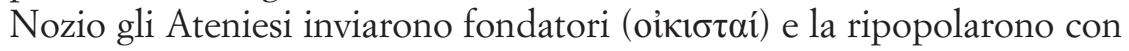
coloni ateniesi, forse cleruchi, e un certo numero di Colofoni da altre poleis $^{70}$. Si riferisce con buona probabilità a questa fase di rifondazione un'iscrizione $^{71}$, in verità piuttosto lacunosa, che attesta l'invio di 5

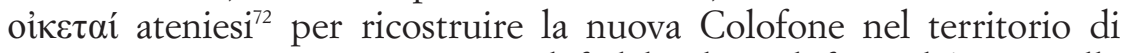
$\mathrm{Nozio}^{73}$ e contiene un giuramento di fedeltà dei Colofoni ad Atene e alla democrazia $^{74}$. Datato in passato al 447 a.C. su base paleografica, il decreto per Colofone è fra quei documenti concernenti la lega ateniese

Oligarchen". In realtà, è impossibile capire se l'intervento dei Persiani sia avvenuto nel tentativo di una delle fazioni di regolare questioni inerenti alla politica interna, rispetto all'orientamento della politeia, se si sia trattato, come ritengo più probabile, di una contrapposizione fra filo-persiani e filo-ateniesi, o se entrambe le componenti sino entrate in gioco. Si può comunque osservare che la compagine che lasciò Colofone per Nozio nel 430 non sembra essere stata tanto compatta: è possibile affermarlo sia a partire dalla ripresa della stasis a Nozio, sia dal fatto che Tucidide (III, 34.4) ricorda la successiva rifondazione di Nozio da parte di coloni ateniesi che ricondussero "tutti i Colofoni dalle città, dovunque ce ne fossero".

69 Sul coinvolgimento di Pissutne nelle vicende di Colofone e Nozio, specchio di una linea politica coerente del satrapo della Lidia verso le città greche, vedi Bearzot 2017, 48-50.

70 Thuc. III, 34.4.

71 IG I3 37.

72 Nell'edizione di $I G \mathrm{I}^{3} 37$ il termine compare con sicurezza nel frammento B

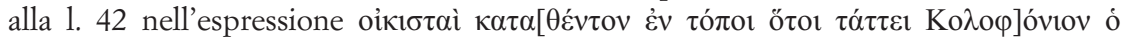
vó $\mu$ os, mentre è integrato con ragionevolezza nel frammento $\mathrm{A}$ alla 1.20 , dove si legge

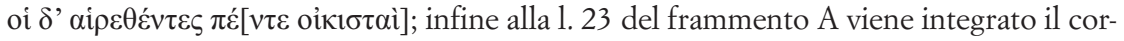

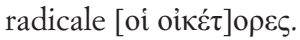

73 Piérart 1984, 171.

74 Datazione tradizionale 447 a.C.: ATL III: 282; Meiggs-Lewis 1969, 124; Parke 1985, 122 (non menziona esplicitamente l'iscrizione, ma ricorda la presunta defezione di Colofone nel 447 a.C.); Low 2008, 71; Rhodes 2008, 505; Bolmarcich 2010, 116 n. 12; la datazione al 427 è sostenuta da Mattingly 1996, 173-178, 373-374; Piérart 1984, 168-171; Rubinstein 2004, 1078; Papazarkadas 2009, 70. 
di $\mathrm{V}$ secolo la cui datazione è stata gravemente condizionata dai criteri adottati dagli editori di $A T L$; ma la revoca in dubbio della correttezza di questi parametri, in particolare della presenza del sigma a tre barre, ha portato a riconsiderare molti documenti epigrafici e a proporre nuove ipotesi di datazione ${ }^{75}$.

La stasis del 427 rappresenta così un contesto adeguato per l'emanazione del decreto sulla rifondazione di Colofone per più di una ragione: la tesi tradizionale doveva supporre che i Colofoni avessero defezionato dalla lega intorno al 448, ma di questa defezione non c'è traccia nelle fonti letterarie; essa si può al massimo postulare a partire dall'assenza di Colofone nelle liste dei tributi ${ }^{76}$; la narrazione di Tucidide e il decreto parlano entrambi di rifondazione del sito di Colofone, senza nominare Nozio; la rifondazione e l'invio di oiketai si addice maggiormente alla riqualificazione di un territorio secondario, come Nozio, in cui si trovavano i Colofoni in esilio; l'intervento di rifondazione ateniese, dunque, identifica una nuova Colofone (probabilmente la città bassa nel territorio di Nozio) ${ }^{77}$, ma riconosce nel contempo la sopravvivenza

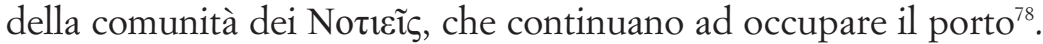

Può essere dunque questa la stasis che Aristotele ha in mente? Non possono esservi certezze al riguardo. Nel 430 la stasis scoppiò per questioni riconducibili all'orientamento politico di due fazioni interne

75 Nonostante la datazione in base alla forma delle lettere possa rappresentare un criterio utile, altri elementi, che risultano meno soggettivi (prosopografia, fraseologia, contesto storico, lingua e grammatica) dovrebbero avere la priorità come criteri di datazione, cf. Tracy 2014, 115.

76 ATL II, 68-69 (testo del trattato); ATL III, 282-284; Meiggs-Lewis 1969, 123-24. La tesi della defezione del 448 si basa anche sulle liste dei tributi, in cui Colofone, che nel periodo dal 454/3 al 451/0 pagava un phoros di tre talenti, risulta assente dalle liste del secondo periodo (dal 450/49 al 447/6 a.C.).

77 È da collegare con questa fase anche il decreto onorifico per Apollonofane di Colofone ( $I G \mathrm{I}^{3}$ 65), datato al 427/6 a.C., onorato per aver mantenuto per gli Ateniesi il forte di Dios Hieron con l'epimeleia degli strateghi e della bulé e la prosodos (11. 14-

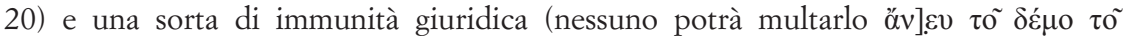
A $\theta \varepsilon v[\alpha i ́ o v, ~ 11.21-22$ con Ma 2009, 127 con paralleli). Per la carriera di Apollonofane vedi ora Brambilla 2019.

78 Sono queste le conclusioni, a mio avviso convincenti, cui è giunto Piérart 1984, 171. L'interpretazione di Piérart supera le obiezioni che erano state mosse a Mattingly sul fatto che la datazione al 427 non si accordasse con il quadro di $I G \mathrm{I}^{3} 37$, in cui Atene sembra avere pieno controllo di Colofone. 
a Colofone; Nozio fu la destinazione degli esuli, che non fecero nulla per tornare in patria. Nel 427 la stasis divampò a Nozio e prese le fila, piuttosto che da una contrapposizione fra Colofone e Nozio, da un dissidio interno alla stessa Nozio, in cui probabilmente si riproposero le stesse dinamiche che avevano innescato la stasis a Colofone pochi anni prima. Benché non si possa escludere che Aristotele pensasse ad un episodio specifico, è un fatto che i dettagli degli eventi collegati con la stasis non coincidono totalmente con l'assunto espresso nel passo. Piuttosto, pare che il filosofo estrapoli dalla storia della città un principio: la vicinanza fra Colofone e Nozio e lo statuto non determinabile di Nozio hanno fatto sì che Colofone abbia potuto estendere la sua influenza su una polis vicina esportandovi la stasis. La topografia, se così letta, diventa un potenziale incentivo al prolungamento anche geografico della conflittualità civica.

\subsection{Atene: la città e il porto}

Com'è noto, la popolazione del Pireo acquista una notorietà particolare nelle fonti per il suo contributo alla causa democratica all'epoca della guerra civile ateniese. Si tratta, però, di un caso isolato, di una ribalta tanto importante quanto momentanea che non conoscerà, stando alle fonti superstiti, occorrenze paragonabili.

La genesi del coinvolgimento del Pireo nella guerra civile è ben nota. I termini della resa nel marzo del 404 a.C. imposero agli Ateniesi la consegna della flotta, con l'eccezione di 12 triremi, e la distruzione delle Lunghe Mura. Qualche settimana dopo aver stretto l'accordo, un Lisandro trionfante entrava in Atene dal Pireo insieme agli esuli. A questo proposito Senofonte ricorda che la demolizione delle Mura del Pireo fu accompagnata dalla musica delle flautiste e dall'entusiasmo di molti che pensavano che "quel giorno segnasse l'inizio della libertà per la Grecia" ${ }^{\text {"9. }}$. Con l'eliminazione delle Mura veniva meno il collegamento fra l'asty e la zona portuale, simbolo della potenza militare ateniese e, soprattutto, sede di quella flotta che ne aveva costituito il principale presupposto. Robert Garland ha sottolineato che l'obiettivo degli Spartani non era solo militare, ma soprattutto politico, perché la separazione fisica fra città e porto avrebbe posto un freno al progresso politico della città o, detto in

79 Xen. Hell. II, 2.23. 
altri termini, avrebbe ostacolato l'ulteriore democratizzazione di Atene ${ }^{80}$. Il progetto politico spartano fu messo in atto dai Trenta, che emanarono una serie di provvedimenti rivolti specificamente al Pireo. L'attenzione nei confronti del Pireo fu improntata fin da subito al ridimensionamento e al controllo militare e amministrativo di quell'area. Lo testimonia certo l'istituzione del collegio dei Dieci di stanza al Pireo, una sorta di magistratura parallela agli Undici, che teneva sotto controllo quell'area ${ }^{81}$. Lo testimoniano, altresì, l'espulsione dall'asty di quanti erano stati estromessi dall'esercizio della cittadinanza attiva e la loro ricollocazione al $\mathrm{Pireo}^{82}$. Se prestiamo fede a Plutarco, la stessa decisione dei Trenta di modificare la disposizione del bema della Pnice, orientato in origine verso il mare, e ora rivolto verso l'interno, ebbe un significato altamente simbolico ${ }^{83}$ e nacque dalla considerazione che l'impero marittimo era stato all'origine della democrazia e che l'oligarchia sarebbe stata più favorevole agli agricolto$\mathrm{ri}^{84}$. Con la redazione della lista dei Tremila, il Pireo divenne zona di confino per gli esclusi, ai quali si aggiunsero con buona probabilità i dissidenti e gli oppositori del regime, almeno nella prima fase del governo dei Trenta. La ricollocazione nel Pireo isolava i democratici, privi di una guida politica e di risorse militari, in una zona dell'Attica che i Trenta si adoperarono a presidiare energicamente. Se da un lato, dunque, l'esilio interno dei democratici al Pireo poteva essere giustificato dal fatto che esso, luogo d'elezione per l'interazione fra gli Ateniesi e gli "altri" ${ }^{5}$, incar-

80 Garland 1987, 33.

81 Pl. Epist. VII, 324d.

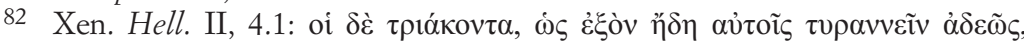

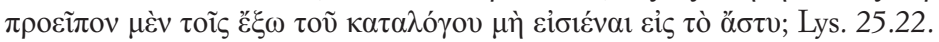

83 Di esempio di "topografia politicamente orientata" e di "uso degli spazi geografici come metafore della metabole tes politeias" ha parlato Daverio Rocchi 2004, 301.

84 Plut. Them. 19.4. Il passo è, in effetti, problematico. La spiegazione di Plutarco è stata messa in dubbio da Moysey 1981, che assegna la ricostruzione della Pnice alla democrazia restaurata, seguito da Hansen 1982, 243 n. 9. Per un approccio meno scettico al passo di Plutarco si veda Garland 1987, 35 e Shear 2011, 177-178. Shear, in particolare, fa riferimento all'esistenza di scavi archeologici che hanno confermato che il luogo deputato alle riunioni dell'Ecclesia fu ricostruito intorno alla fine del $\mathrm{V}$ secolo e che l'orientamento della struttura fu rovesciato. Tuttavia, è difficile ammettere che i Trenta, nella difficile situazione economica che la città sperimentava, abbiano deciso di investire in un'opera che non avrebbero mai usato.

85 Per questo genere di interazione, vista dai detrattori della democrazia come una mescolanza con individui di condizione sociale differente, vedi Loddo 2019, 79-80. 
nava meglio di qualunque altra zona della città i valori della democrazia radicale e rappresentava il più chiaro contraltare al pensiero oligarchico ${ }^{86}$, dall'altro finì per essere una enclave rigidamente controllata dal nuovo regime. Privato delle Lunghe Mura, il Pireo ripristinava idealmente quella separazione fisica rispetto alla città, quella condizione di perea, cui Strabone allude riferendosi a un passato lontano, ma che doveva essere ancora evidente nel $\mathrm{V}$ secolo, alla vista dell'esigua striscia di terra che lo univa al resto dell'Attica ${ }^{87}$.

Se pensiamo al fatto che il Pireo divenne la sede dei democratici durante la guerra civile, non possiamo non concludere che Aristotele ha in mente la stasis del 404/3 quando parla della contrapposizione fra il porto e la città. Ma è davvero questa la funzione dell'esempio nel passo?

\section{LA FUNZIONE DELL'EXEMPLUM RELATIVO AD ATENE}

Si è detto che parte della critica ha ritenuto che le coppie Clazomene-Chito e Colofone-Nozio, che Aristotele ha utilizzato per esemplificare il suo assunto, non si riferiscano a episodi specifici, ma evochino casi di comunità civili in cui la stasis appariva qualcosa di endemico. Solo nel caso di Atene Aristotele avrebbe in mente un episodio concreto e l'identificazione più probabile è stata trovata nelle conseguenze della guerra civile che scoppiò ad Atene quando i Trenta stabilirono il loro regime ${ }^{88}$. È probabilmente la formulazione piuttosto generica dell'affermazione di Aristotele che rende possibile ricorrere a questo argomento. Mi pare, però, che l'esempio relativo ad Atene funzioni diversamente dai primi due e che, in definitiva, Aristotele lo usi per illustrare un principio differente, benché correlato con i precedenti.

Nel commentare l'uso da parte di Aristotele di esempi storici per spiegare un aspetto specifico nella stasis, Andrew Lintott ha sottolineato che il trattamento di Aristotele di questi episodi risulta spesso insoddisfacente, poiché manca un'analisi estesa dei contesti in cui la stasis ha avuto origine e un'adeguata considerazione degli altri fattori che hanno

\footnotetext{
86 Garland 1987, 33-35.

87 Strab. I, 3.18. Cfr. Garland 1987, 7; Carugati 2019, 179 ss.

88 Schütrumpf-Gehrke 1996, 445; De Luna 2016, 321.
} 
cooperato a inasprire la faziosità nella città ${ }^{89}$. Allo stesso tempo gli esempi funzionano come istantanee di un evento specifico che non includono informazioni sul momento in cui la stasis scoppia.

Se rivolgiamo l'attenzione alla struttura del nostro passo, possiamo notare che l'affermazione sulle differenze in merito all'appartenenza politica del Pireo e della città non pare collocata in un momento specifico, ma viene presentata come una sorta di assioma, la cui validità sembra essere generale. L'uso del tempo presente (cioív) può indicare il tempo da cui si osserva un'azione - come appariva la popolazione del Pireo al tempo in cui Aristotele scriveva - o lo stato dell'azione nel suo aspetto durativo o continuativo - come appare in generale la popolazione del Pireo rispetto a quella della città. A me pare che il secondo aspetto sia prevalente. A ben vedere, il ragionamento di Aristotele è articolato secondo una struttura chiastica, che prevede, dapprima, l'enunciazione di un primo principio teorico, seguito dal primo exemplum, poi, la menzione di un secondo exemplum, seguito dal secondo principio teorico, questa volta preceduto e arricchito da un paragone:

1.

a. Primo assunto teorico: differenze nella topografia della città sono causa potenziale di contrasti interni e staseis

b. Primo exemplum: così è avvenuto a Clazomene, dove quelli dell'isola sono in contrasto con quelli che abitano la perea, e a Colofone, dove la stasis si è allargata alla polis di Nozio

2.

$b^{1}$. Secondo esempio: Anche ad Atene gli abitanti della zona portuale hanno interessi e visioni politiche differenti rispetto a chi abita nella zona della città

$a^{1}$. Paragone e secondo assunto teorico: Come in guerra gli attraversamenti dei canali, anche di quelli assai stretti, rompono i ranghi delle falangi, così sembra che ogni differenza produca un contrasto

La struttura chiastica $a-b-b^{1}-a^{1}$ rende evidente che nel caso di Atene non c'è un riferimento né preciso né esplicito a una stasis concreta, sebbene chi conosce la storia ateniese non possa non pensare alla

89 Lintott $2013^{2}, 241$. 
guerra civile del 404/3. Mi pare però che il punto per cui Aristotele introduce il caso ateniese sia solo tangenzialmente collegato con l'assunto generale - la stasis nelle città può essere cagionata dalle condizioni del territorio quando queste impediscono l'unità territoriale; l'esempio ateniese è piuttosto un sotto-caso da considerare nel quadro delle conseguenze provocate da una differenza nella topografia della città, che sfocia in una polarizzazione nell'orientamento politico ed è riconoscibile nelle scelte abitative della popolazione. In effetti, mentre i primi due casi hanno a che fare con una polis che si sdoppia, che guarda a un territorio in qualche modo dipendente come a un pezzo della città fuori dalla città - il territorio (chora) della città, in effetti, non coincide con una sola polis (mia polis) - Atene è una polis unitaria dal punto di vista territoriale ${ }^{90}$, ma determinante perché Aristotele la prenda in considerazione in questo contesto risulta la diversificazione dell'orientamento politico dei suoi abitanti. Potremmo dire, per semplificare, che il discorso scivola, come testimonia il cambio di soggetto nel passo, dalla polis che si sdoppia alla popolazione che si divide, pur continuando ad occupare il territorio di un solo stato. ${ }^{91} \mathrm{E}$ infatti già Newman notava che

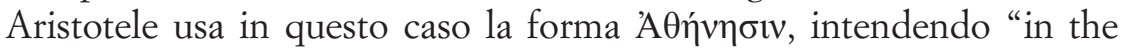
Athenian State" e includendo così il Pireo".

Ad escludere la possibilità che il filosofo abbia in mente un episodio preciso concorre il ricorso al paragone, inserito quale ausilio interpretativo dell'assunto teorico che segue. L'idea che ogni differenza produce un contrasto è paragonata all'attraversamento dei canali da parte della falange oplitica. Quando, in tempo di guerra, gli opliti si trovavano ad attraversare torrenti, canali, corsi d'acqua dovevano affrontare difficoltà oggettive come la natura irregolare, ineguale, del terreno, stare attenti alle correnti, specie quando dovevano transitare sui fiumi, evitare di inciampare, scivolare o ferirsi con ciottoli e detriti. Soprattutto lo schieramento

90 È chiaro che il discorso di Aristotele esula dalla questione delle cleruchie.

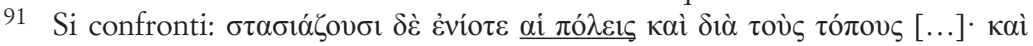

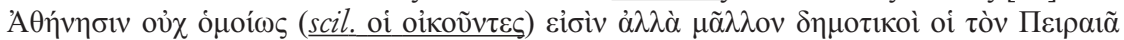

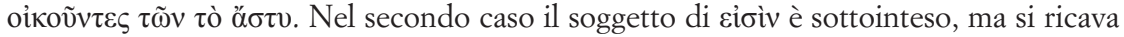
agevolmente dal prosieguo della frase. Devo al confronto con Elena Langella questo punto e l'osservazione, giunta per litteram e che riporto verbatim, che nella seconda frase "l'abitare è comune, mentre quello che cambia - e che caratterizza in posizione attributiva gli abitanti - è il fattore geo-/topografico".

92 Newman 1902, 317. 
tipico della falange, ordinato e serrato, finiva per scomporsi. È a questo stato di cose che Aristotele sembra riferirsi: come l'attraversamento di un canale, apparentemente insignificante, era in grado di rompere i ranghi della falange, così ogni differenza, per quanto minima, poteva essere la causa di divisione e conflitto $(\delta i \alpha ́ \sigma \tau \alpha \sigma ı)^{93}$. Se il ragionamento è corretto, se ne può dedurre che l'esempio ateniese è introdotto per illustrare questo secondo principio generale.

L'analisi fin qui condotta trova ulteriore conferma se si guarda ai termini in cui viene presentata l'opposizione. Questo è il punto più importante da valutare per capire se Aristotele abbia menzionato qui un evento specifico. Infatti, se Aristotele avesse voluto evocare la situazione politica sorta al tempo dell'oligarchia dei Trenta, avrebbe dovuto parlare di uno scontro tra democratici e oligarchici. L'espressione

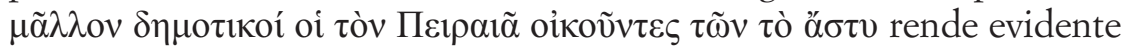
che l'opposizione cui si riferisce concerne i sostenitori della democrazia radicale e i partigiani di una forma più moderata di democrazia. È altamente improbabile, dunque, che Aristotele possa riferirsi ai Trenta o ai loro sostenitori come moderatamente democratici. La sua affermazione presuppone l'esistenza di una diversificazione nell'attaccamento dei cittadini alla democrazia a seconda del luogo di residenza: chi vive più vicino al mare sarebbe più democratico, mentre chi vive in città avrebbe un orientamento meno democratico. Si tratta di una sorta di regionalismo politico che corrisponde a una dicotomia socio-economica. In altre parole, questo giudizio combina l'analisi dell'appartenenza politica con la valutazione dello status economico delle componenti sociali.

Com'è noto, Aristotele non è estraneo a spiegare il conflitto civico attraverso categorie socio-politiche e divisioni territoriali. Nel descrivere la lotta per l'arcontato tra le fazioni negli anni successivi alle riforme di Solone, egli accoglie parzialmente la lettura, di derivazione erodotea, che collegava le fazioni con i nomi delle regioni in cui i loro capi possedevano e coltivavano le loro terre ${ }^{94}$, aggiungendo alla divisione territoriale così concepita uno specifico orientamento politico ${ }^{95}$. Non ci interessa

93 Sull'uso di questo termine cfr. Newman 1902, 318.

94 Hdt. I, 59.3.

95 Arist. Ath. Pol. 13.4. Cf. Plut. Sol. 13.1-3; 29.1; Amat. 673d; Prae. ger. reip. 805d-e. Sulle differenze nei resoconti di Ath. Pol. e Plutarco si veda Rhodes 1981, 179, 185-186. 
qui valutare l'adeguatezza della sua costruzione ${ }^{96}$, ma si vuole sottolineare che questo tipo di analisi, espressa in termini di regionalismo sociopolitico, è caratteristica di Aristotele o quantomeno della sua scuola.

In un frammento della Costituzione dei Nassi, nel commentare il

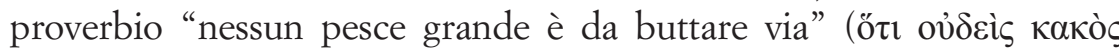

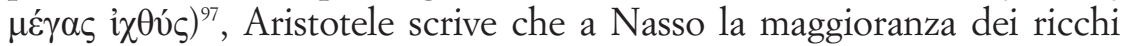

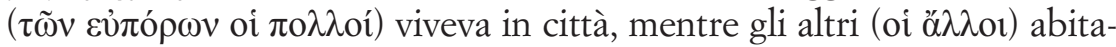

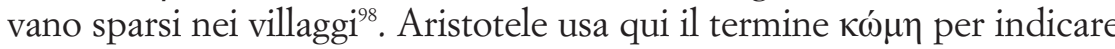
i villaggi, ma è probabile che lo intenda come sinonimo dorico di $\delta \tilde{\eta} \mu \varsigma^{99}$. Dal prosieguo del frammento, che riguarda un episodio di stasis, appare chiaro che oi ó $\lambda \lambda$ or sono il demos nella cornice di una ristretta oligarchia. Infatti la stasis a Nasso scoppiò quando Telestagora, probabilmente un notabile che viveva in campagna e agiva da patrono del demos, fu assalito da un gruppo di giovani nobili ubriachi ${ }^{100}$. Ne scaturì una rivolta popolare, guidata da Ligdami, il futuro tiranno dell'isola ${ }^{101}$. La fine dell'oligarchia fu dovuta all'alleanza tra la nobiltà terriera e i commercianti che vivevano nell'area della città vicina al mare. È da notare che il villaggio dove viveva Telestagora e da cui partì la rivolta, Lestadai, il cui nome significa "borgo di ladri", era situato vicino al mare e che la composizione della sua popolazione ricorda quella del Pireo.

Una conferma del fatto che l'affermazione di Aristotele sulle divisioni politiche tra il Pireo e l'asty ha un carattere generale e non si riferisce alle lotte civili sotto i Trenta viene dall'analisi del termine

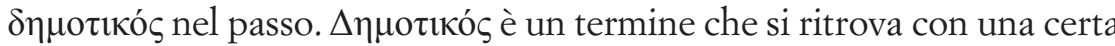
frequenza nelle opere aristoteliche, in particolare in quelle politiche (Politica e Athenaion Politeia) ${ }^{102}$. Se si limita l'indagine alla Politica, con l'obiettivo di valutare il modo in cui Aristotele usa il termine in quest'o-

96 Per una valutazione adeguata si rimanda a Hopper 1961; Rhodes 1981, 184-187.

97 Cf. la traduzione di Huxley 1973, 273: "no big fish is a rotten one".

98 Arist. fr. 558 Rose = Ath. VIII, 40.348b-c.

99 Arist. Poet. 1448a 35-37.

100 Sulla vicenda si rimanda a Costa 1996, 155-170.

101 Sulla presa del potere da parte di Ligdami vedi Arist. Pol. V, 6.1305a 37-40.

102 Arist. Ath. Pol. 6.2-3; 9.1; 10.1; 13.4-5; 14.1; 16.8; 18.5; 22.1; 27.1; 29.3;34.3; 41.2; Pol. II, 6.1266a 22; II, 7.1266b 22; II, 11-12.1273b 13, 41; IV, 3.1290a 28; IV, 5.1292b 13; IV, 14.1298a 10; IV, 14.1298b 18; IV, 15.1299b 32-37; IV, 15.1300a 32; IV, 16.1301a 11; V, 3-4.1303b 11, 36; V, 5.1305a 28; V, 8.1308a 11; V, 9.1309b 20; V, 9.1310a 17; VI, 1.1317a 19; VI, 2.1317b 3, 11, 30, 40; VI, 3.1318a 18-19; VI, 4.1319b 28; VI, 5.1320a 2, 14, 33; VI, 7.1321a 14; VI, 8.1323a 4; Metaph. 989a. 
pera, è possibile affermare che egli impiega $\delta \eta \mu$ o๘ıќ́ natura democratica del governo ${ }^{103} \mathrm{o}$ di alcune istituzioni specifiche, come il Consiglio, o per riferirsi a concetti tipicamente democratici ${ }^{104}$; per segnalare un membro della classe popolare (i poveri) o ciò che ha un carattere popolare ${ }^{105}$; per indicare coloro che appartengono alla fazione democratica (opponendoli a gnorimoi) ${ }^{106}$. Non è sempre facile distinguere chiaramente quale di queste sfumature concettuali prevalga, poiché in alcuni casi la sfumatura politica può coesistere con il significato socio-economico, che si riferisce all'estrazione sociale. Per esem-

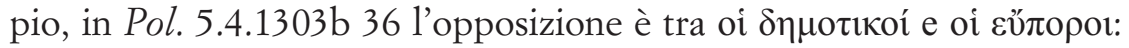
in questo caso la contrapposizione politica si esprime secondo categorie socio-economiche, ma implica anche l'appartenenza a fazioni politiche antagoniste.

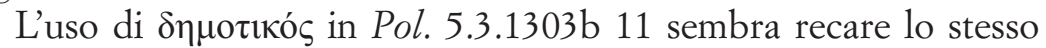
duplice significato, in quanto si riferisce all'affiliazione a una fazione politica come conseguenza diretta dell'appartenenza a un gruppo sociopolitico ${ }^{107}$. Ma, come è stato notato, Aristotele qui non ha in mente solo i cittadini ateniesi di pieno diritto. La stessa formulazione dell'espres-

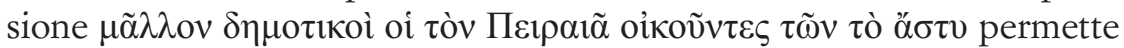
di sostenere che gli individui di cui si parla non sono solo gli Ateniesi katharoi, in quanto l'uso del verbo oíkź $\omega$ può riferirsi sia ai cittadini che ai non-cittadini. In particolare, si può suggerire che anche i meteci e gli schiavi siano inclusi nella categoria che Aristotele ha in mente poiché oíkś $\omega$, quando usato intransitivamente, indica la residenza degli stranieri in un demo specifico ${ }^{108}$. Ciò significa che è il criterio della residenza piuttosto che la nascita a guidare l'osservazione di Aristotele. Nel tentativo di verificare l'attendibilità dell'affermazione di Aristotele circa il maggiore attaccamento alla democrazia degli abitanti del Pireo, James Roy ha richiamato l'attenzione sul fatto che è impossibile rintracciare

103 Arist. Pol. II, 7.1266b 22; IV, 3.1290a 28; V, 9.1310a 17; VI, 5.1320a 2.

104 Arist. Pol. II, 11.1273b 13, 41; IV, 14.1298a 10; IV, 15.1299 b 32-37; IV, 15.1300a 32; IV, 16.1301a 11; V, 9.1309b 20; VI, 2.1317b 3, 30, 40; VI, 3.1318a 18; VI, 4.1319b 28; VI, 7.1321a 14.

105 Arist. Pol. II, 6.1266a 22; 1298b 18; VI, 1.1317a 19.

106 Arist. Pol. V, 4.1303b 36; V, 5.1305a 28; V, 8.1308a 11; VI, 2.1317b 11; VI, 3.1318a 19; VI, 5.1320a 14, 33.

107 Von Reden 1995; Roy 1998.

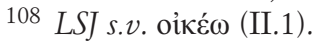


precise tendenze politiche tra i residenti del Pireo ${ }^{109}$. Per quanto riguarda la popolazione cittadina del Pireo, le cifre che si possono dedurre dai calcoli fatti a partire dalla quota buleutica non sono affidabili, perché non prendono in considerazione tutti quegli individui che, pur vivendo al Pireo, mantenevano la loro residenza in un altro demo. Per quanto riguarda la popolazione metecica, la documentazione relativa a 366 stranieri residenti permette di concludere che c'era una maggiore concentrazione di meteci nell'asty che nel Pireo. Inoltre, Roy ha osservato che la popolazione metecica del Pireo non sembra differire da quella dell'asty da un punto di vista socio-politico ${ }^{110}$. Queste considerazioni porterebbero a concludere che i dati disponibili non corroborano l'affermazione di Aristotele sulla natura di mallon demotikoi di coloro che vivono al Pireo.

Ma il passo non può essere liquidato sulla base di questo presupposto. Ancora Roy ha affermato che la stasis di cui parla Aristotele deve essere intesa non come aperta ostilità politica, ma come persistente tensione socio-politica. Le parole di Aristotele suggeriscono che almeno una parte della cittadinanza credeva che tra il Pireo e la città esistesse una reale tensione sulla base della convinzione che i cittadini del Pireo fossero più demotikoi. Queste convinzioni non sono isolate, ma emergono in altri testi relativi al Pireo in un discorso in cui il porto è frequentemente associato con la flotta e criticato per questo motivo più o meno esplicitamente. Si possono richiamare a questo proposito alcuni passi in cui si sottolinea il legame tra il Pireo e la flotta. In primo luogo, in Pol. 4.1291b 17-25 Aristotele, partendo dal presupposto che sia la fazione popolare sia quella dei notabili possono avere una composizione sociale eterogenea, menziona coloro che hanno a che fare con il mare e identifica tra questi i marinai del Pireo. Il passo riecheggia un dibattito su democrazia, flotta e potere navale che richiama non solo il punto di vista polemico del Vecchio Oligarca sulla democrazia ateniese ${ }^{111}$, ma anche ciò che Aristotele dice nel Libro 6 della Politica: mentre una grande disponibilità di opliti favorisce l'instaurazione dell'oligarchia, poiché gli opliti sono più spesso di condi-

109 Roy 1998, 194-195.

110 Roy 1998, 196-197.

111 Xen. [Ath. Pol.] 1.2. Per un'analisi della posizione dell'anonimo autore della Costituzione degli Ateniesi del V secolo sul rapporto fra democrazia e talassocrazia si veda Ceccarelli 1993, 446-451. 
zione agiata che indigenti, la democrazia fiorisce dove crescono le forze vitali della fanteria e della marina ${ }^{112}$. Da ciò deriva un contrasto di classe tra ricchi e poveri che è uno degli elementi chiave della lettura socio-politica di Aristotele, anche se qui non è esplicitamente dichiarato. Nella visione di Aristotele c'è infatti una precisa correlazione tra appartenenza politica, posizione sociale e status occupazionale. Con una visione solo apparentemente deterministica Aristotele continua dicendo che se una lotta civile scoppia in un'oligarchia dove c'è un gran numero di marinai, tale oligarchia può facilmente soccombere ${ }^{113}$.

In secondo luogo, si deve menzionare il luogo classico delle Leggi di Platone in cui l'Ateniese, nel tentativo di identificare le condizioni ideali per la fondazione di una nuova città, chiede al suo interlocutore se tale città debba essere costruita vicino al mare o in una zona interna. Clinia risponde che la città ideale dovrebbe sorgere a 80 stadi dal mare e avere buoni porti ${ }^{144}$. Tuttavia, l'Ateniese ha una visione negativa della vicinanza della città al mare, poiché, incoraggiando il commercio con l'esterno, istilla negli abitanti abitudini discutibili, come la tendenza a ricorrere all'inganno, e diffonde un sentimento di ostilità fra i cittadi$\mathrm{ni}^{115}$. Sebbene l'affermazione non sia riferita espressamente alle democrazie e sembri avere piuttosto una valenza generale, si può aggiungere che Platone individua ripercussioni negative per le città che si affidano alla flotta anche in rapporto al modo di combattere: se le battaglie terrestri incentivano i soldati a mostrare il proprio valore, il combattimento navale incoraggia la codardia, dal momento che la disponibilità delle navi rende possibile la fuga e l'abbandono dello scenario dello scontro $^{116}$. Il ragionamento può valere tanto sul piano individuale sia in relazione ai marinai come gruppo sociale.

112 Arist. Pol. VI, 7.1321a 12-14.

113 Arist. Pol. VI, 7.1321a 14-16.

114 Pl. Leg. IV, 1.704b. Cfr. Pl. Resp. 370e-371b in cui, nonostante l'enfasi sull'importanza dell'autarchia per la città, si dice che tanto l'importazione quanto l'esportazione dei beni di consumo sono ugualmente necessarie.

115 Pl. Leg. IV, 1.705b con Günther 2016, 118-121. Platone esprime una visione simile altrove (Cri.113c-119e), quando sostiene che una città che basi il suo potere sul commercio e sul traffico marittimo è destinata all'estinzione a causa il progressivo e inevitabile prevalere dell'bybris.

116 Pl. Leg. IV, 1.706c. L'idea è accolta da Arist. Pol. IV, 3.1289b; IV, 4.1291b 225; IV, 6.1292b 22-1293a 10; VI, 7.1321a 5-14, ma in maniera meno rigida. Cf. Ceccarelli 1993, 455-457. Per Aristotele (Pol. VII, 6.1327a 11-40) è la necessità che il 
In aggiunta, si può richiamare l'attenzione sul fatto che l'etichetta di demotikos può includere considerazioni sulla coincidenza tra lo stile di vita degli individui e la loro appartenenza a un gruppo socio-politico determinato con specifici interessi di classe. In altre parole, un individuo è demotikos perché esibisce abitudini tipiche della democrazia, secondo un modo di ragionare che appare tipico di Aristotele. Nel libro 4 della Politica egli sostiene che a volte un regime che non è democratico secon-

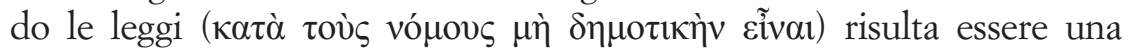

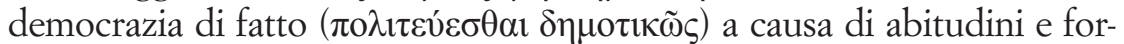

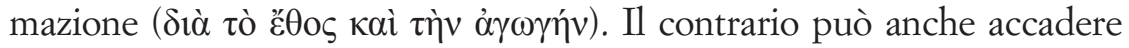
quando una democrazia è governata in modo oligarchico a causa dell'e-

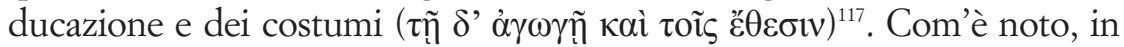
questa sezione della Politica Aristotele si riferisce a quei cambiamenti nello stile di vita della cittadinanza che seguono un cambiamento costituzionale, ma è l'enunciazione del principio a rilevare per il nostro discorso. Il filosofo, in effetti, considera il modo migliore per conservare una politeia il fatto che i cittadini sono educati in un modo che risulti congruente con l'ordinamento della città. Così, egli sostiene:

"Ma il mezzo più importante di tutti quelli menzionati per assicurare la sussistenza di una costituzione, e che oggi da tutti è trascurato, è l'educazione ai fini della costituzione. Infatti le leggi più utili e approvate da tutti i membri della città non saranno di nessuna utilità, se i cittadini non saranno stati abituati ed educati come la costituzione richiede, cioè democraticamente se le leggi sono democratiche, oligarchicamente se le leggi sono oligarchiche, perché quando non sa obbedire il singolo, non lo sa neppure la città nel suo complesso" ${ }^{118}$.

Sembra, pertanto, che questa sia la giusta cornice in cui collocare la relazione tra regime politico, orientamento politico individuale e stile di vita. La teoria aristotelica sembra sviluppare quanto appare embrionalmente, almeno a giudicare dai frustuli superstiti, nel pensiero di un autore come Teopompo, che lega l'orientamento e il destino della poli-

porto sia uno spazio economico regolamentato dal punto di vista giuridico così da rappresentare un vantaggio per la città e non un motivo di tensione. Cf. Günther 2016, 121-122.

117 Arist. Pol. IV, 5. 1292b 11-17.

118 Arist. Pol. V, 9.1310a 12-18. 
teia allo stile di vita della comunità civica o degli individui che detengono il potere ${ }^{119}$. In questa categoria rientrano alcuni frammenti che menzionano il tema della tryphé, tema che anche Aristotele mostra di considerare sia nei frammenti sia nella Politica ${ }^{120}$. Probabilmente la testimonianza più vicina al punto di vista aristotelico viene dal frammento sulla democrazia di Bisanzio e Calcedone.

Ci sono tra l'altro gli abitanti di Bisanzio e di Calcedonia, dei quali Teopompo dice: «Gli abitanti di Bisanzio erano sfrenati e avvezzi ad amoreggiare e a bere nelle bettole, e questo perché per lungo tempo ebbero un regime democratico, e inoltre la città era situata in una zona di scalo commerciale, e quindi tutta la gente passava il tempo tra il mercato e il porto; i Calcedoni, prima di accogliere la loro stessa forma di governo, avevano nella loro totalità abitudini e un regime di vita migliori; quando poi ebbero assaggiato la democrazia dei Bisanzi, si lasciarono corrompere dalla passione per i piaceri, facendosi da temperantissimi e moderatissimi nella vita quotidiana, beoni e prodighi $\gg^{121}$.

Ho già trattato di questo passo altrove ${ }^{122}$. Qui mi limito a sottolineare due degli elementi che rilevano per il nostro discorso. Innanzitutto, è interessante il quadro che Teopompo offre di Bisanzio democratica, in cui il legame fra democrazia e stile di vita si alimenta vicendevolmente: l'ubicazione in uno scalo commerciale; le abitudini dei cittadini che trascorrono le giornate tra il porto e l'agora; l'assuefa-

119 Theopomp. FGrHist 115 FF 62, 105, 114, 233, 281.

120 Arist. F 515 a Rose = Ath. XIV, 10.618c6-619a2 (morte violenta del poeta Teodoro, di cui si dice che era dedito alla tryphé); F 584 Rose = Ath. XII, 25.523c4-d3 (fondazione di Siri da parte di coloni da Colofone e diffusione della tryphé); Pol. II, 6.1265a 28-38 (la tryphé è l'opposto della vita temperante, cf. VII, 5.1326b 30-39); II, 7.1266b 24-26 (l'eccessiva ricchezza porta alla tryphé; cf. II, 9.1269b22-24: lo stile di vita delle donne a Sparta; IV, 15.1300a 7-8: le donne nelle oligarchie amano il lusso); IV, 11.1295b1-1296a9 (l'idea che la tryphé appresa fin dall'infanzia abitua a non ubbidire è inserita nella riflessione sulla condizione economica dei cittadini nella costituzione ideale); V, 1310a 19-25 (adeguare il modo di vivere alla costituzione non vuol dire fare come si vuole: vi rientra l'esempio dei figli dei magistrati nelle città oligarchiche che si danno alla tryphé); V, 10.1311a 8-15 (la relazione fra tirannide, ricchezza e tryphé); V, 10.1312a 8-14 (i philoi del tiranno lo disprezzano perché che conduce una vita improntata alla tryphé e spesso per questo organizzano congiure contro di lui). Uno studio d'insieme delle testimonianze aristoteliche sulla tryphé è in Pezzullo 2012.

121 Theopomp. FGrHist 115 F 62 = Ath. XII, 32.526d-f.

122 Loddo 2016, 192-193. 
zione alla democrazia, che a Bisanzio è stata adottata da molto tempo; la sfrenatezza dei costumi. In secondo luogo, la capacità del modello democratico di trasformare le abitudini di un popolo. La facoltà della democrazia, non è dato sapere se imposta o volontariamente adottata, di corrompere i costumi dei Calcedoni è affermata con forza specie nell'immagine della caduta verso uno stile di vita eccessivamente raffi-

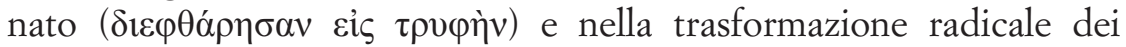
costumi di un popolo, che assapora come ad un banchetto i piaceri del vivere democratico ${ }^{123}$.

\section{CONCLUSIONI}

Ha affermato giustamente Contogiorgis che la conformazione del territorio è fra le cause oggettive dei movimenti rivoluzionari ${ }^{124}$. L'analisi condotta in queste pagine ha mostrato che l'esplosione del conflitto civico può ricondursi solo parzialmente alle caratteristiche fisiche del territorio che ospita la comunità dei cittadini, nel senso che il territorio può impedire la coesione sociale e l'unità politica solo in presenza di altri fattori più prettamente politici. La conformazione geografica, quindi, può diventare "causa oggettiva" della stasis, ma è, in effetti, solo un fattore potenziale di conflittualità, che si attiva laddove i cittadini politicizzano la configurazione dello spazio civico. Ciò non significa sminuire il paradigma che Aristotele ha ricostruito o mettere in discussione la sua conoscenza delle vicende delle città scelte come casi esemplificativi: da un lato, corrisponde al vero che i centri secondari possono aver svolto un ruolo fondamentale nel prolungamento del conflitto civico, perché la stasis può continuare anche dopo il trasferimento o l'allontanamento forzato di una parte della popolazione in questi luoghi; dall'altro è piuttosto la conoscenza complessiva della storia delle città, ad alcune delle quali il filosofo aveva dedicato studi specifici per la sua raccolta di politeiai, ad aver fatto maturare ad Aristotele questa convinzione.

L'indagine ha mostrato che Aristotele, nella costruzione del trittico di casi impiegati per esemplificare il funzionamento della stasis dia

123 Loddo 2016, 193.

124 Contogiorgis 1978, 184. 
tous topous, non si riferisce a un episodio di stasis in particolare. Non solo è difficile connettere con certezza i casi di Clazomene/Chito e Colofone/Nozio con un unico, specifico momento di guerra civile, ma l'esempio ateniese sembra avere rivestito un'altra funzione. Mentre i primi due esempi sono quelli che Aristotele ha scelto come i più adatti ad illustrare il principio che la stasis può essere favorita da una conformazione geografica del territorio che ostacola l'unità della polis, il riferimento ad Atene esemplifica la situazione in cui il carattere diseguale e disomogeneo della popolazione in termini di sentimento politico si riverbera nelle scelte insediative. L'interesse della sua riflessione non risiede tanto nella correttezza della ricostruzione, quanto nel fatto che nel proporla Aristotele ci ha fornito un primo esempio di demografia politica, appendice della riflessione teorica più ampia su urbanistica e costituzione ideale ${ }^{125}$, applicata allo studio della città antica.

\section{ABBREVIAZIONI USATE}

$A T L=$ B.D. Meritt, H.T. Wade-Gery, M.F. McGregor (eds.), The Athenian Tribute Lists, voll. II-III, Princeton 1949-1950.

$G H I=$ P.J. Rhodes, R. Osborne, Greek Historical Inscriptions Edited with Introduction, Translations, and Commentaries, voll. I-II, Oxford 2003-2017.

125 Su questo aspetto cfr. ora lo studio di Carsana-Zizza 2019. 


\section{BIBLIOGRAFIA}

Amit 1961 - M. Amit, Le Pirée dans l'histoire d'Athènes à l'époque classique, in BAGB, n. 20.4, Paris, Les Belles Lettres.

Aubonnet 1973 - J. Aubonnet (a cura di), Aristoteles. Politique, Paris, Les Belles Lettres.

Bearzot 1994 - C. Bearzot, Esilii, deportazioni, emigrazioni forzate sotto regimi non democratici, in M. Sordi (a cura di), Emigrazione e immigrazione nel mondo antico, CISA 21, Milano, Vita e Pensiero.

Bearzot 2004 - C. Bearzot, Federalismo e autonomia nelle Elleniche di Senofonte, Milano, Vita e Pensiero.

Bearzot 2017 - C. Bearzot, Pissutne, satrapo della Lidia, in RaRe, n. 9, Roma, Tored.

Bearzot 2020 - C. Bearzot, Extradition et saisie de la personne des réfugiés athéniens à l'époque des Trente Tyrans, in Pallas, n. 112, Toulouse, Presses Universitaires du Mirail.

Bettalli 2012 - M. Bettalli, Guerra e violenza a Corinto, 392 a.C. (Senofonte, Elleniche, IV, 4, 1-13), in S. Cataldi - E. Bianco - G. Cuniberti (a cura di), Salvare le poleis, costruire la concordia, progettare la pace, Alessandria, Dell'Orso.

Bolmarcich 2010 - S. Bolmarcich, Communal Values in Ancient Diplomacy, in R.M. Rosen - I. Sluiter (a cura di), Valuing Others in Classical Antiquity, Leiden Boston, Brill.

Brambilla 2019 - A. Brambilla, Migrants, Warfare, and Social Promotion in Classical Greece, in RaRe, n. 13, Roma, Tored.

Brill 2020 - S. Brill, Aristotle on the Concept of Shared Life, Oxford, Oxford University Press.

Buckler 2003 - J. Buckler, Aegean Greece in the Fourth Century BC, Leiden - Boston, Brill.

Cairns, Canevaro, Mantzouranis 2021 - D. Cairns, M. Canevaro, K. Mantzouranis, Aristotle on the Causes of Civil Strife: Subjective Dispositions, Proportional Justice and the 'Occasions' of Stasis, in Maia, n. 73.3, Bologna, Cappelli.

Carsana - Zizza 2019 - C. Carsana - C. Zizza, "¿ Dónde fundaremos nuestra ciudad?" Lugares y constituciones ideales en Platón, Aristóteles y Cicerón, in DHA, n. 45.2, Besançon, Presses Universitaires de Franche-Comté.

Carugati 2019 - F. Carugati, Creating a Constitution: Law, Democracy, and Growth in Ancient Athens, Princeton - Oxford, Princeton University Press.

Ceccarelli 1993 - P. Ceccarelli, Sans thalassocratie, pas de démocratie? Le rapport entre thalassocratie et démocratie à Athènes dans la discussion du Ve et IVe siècle av. J.C., in Historia, n. 42.4, Stuttgart, Steiner.

Contogiorgis 1978 - G.D. Contogiorgis, La théorie des révolutions chez Aristote, Paris, R. Pichon et R. Durand-Auzias.

Costa 1996 - V. Costa, Ligdami, Pisistrato e la fondazione della tirannide nassia, in E. Lanzillotta - D. Schilardi (a cura di), Le Cicladi e il mondo egeo (Seminario internazionale di Studi, Roma, 19-21 novembre 1992), Roma, Università degli studi di Roma 'Tor Vergata'.

Costanzi 2020 - M. Costanzi, Mobility in the Ancient Greek World: Diversity of Causes, 
Variety of Vocabularies, in F. De Angelis (a cura di), A Companion to Greeks across the Ancient World, Hoboken, Wiley.

Daverio Rocchi 2004 - G. Daverio Rocchi, Forme urbane e rappresentazioni geografiche della politeia, in S. Cataldi (a cura di), Poleis e politeiai. Esperienze politiche, tradizioni letterarie, progetti costituzionali (Atti del Convegno Internazionale di Storia greca, Torino 29-31 maggio 2002), Alessandria, Dell'Orso.

De Luna 2016 - M.E. De Luna, in M.E. De Luna - C. Zizza - M. Curnis (a cura di), Aristotele. Politica. Libri V e VI, Roma, L'Erma di Bretschneider.

De Luna 2020 - M.E. De Luna, Dai luoghi alla stasis e viceversa. Considerazioni su un passo di Aristotele, in RSA, n. 50, Bologna, Pàtron.

Duplouy 2013 - A. Duplouy, Les Mille de Colophon. Totalité symbolique d'une cite de Ionie (Vie - Iie s. av. J.-C.), in Historia, n. 62.2, Stuttgart, Steiner.

Fogazza 1974 - G. Fogazza, Colofone arcaica, in QUCC, n. 18, Pisa, Serra.

Fornis 2006 - C. Fornis, La ficticia unión entre Corinto y Argos (392-386 aC), in MediterrAnt, n. 9.2, Pisa, Istituti Editoriali e Poligrafici Internazionali.

Forsdyke 2005 - S. Forsdyke, Exile, Ostracism, and Democracy. The Politics of Expulsion in Ancient Greece, Princeton, Princeton University Press.

Garland 1987 - R. Garland, The Piraeus: From the Fifth to the First Century B.C., London, Duckworth.

Garland 2014 - R. Garland, Wandering Greeks: The Ancient Greek Diaspora from the Age of Homer to the Death of Alexander the Great, Princeton - Oxford, Princeton University Press.

Gehrke 1985 - H.-J. Gehrke, Stasis: Untersuchungen zu den inneren Kriegen in den griechischen Staaten des 5. und 4. Jahrbunderts v. Chr., München, Beck.

Giangiulio 2018 - M. Giangiulio, Oligarchies of 'Fixed Number' or Citizen Bodies in the Making?, in R. Brock - A. Duplouy (a cura di), Defining Citizenship in Archaic Greece, Oxford, Oxford University Press.

Griffith 1950 - G.T. Griffith, The Union of Corinth and Argos (392-386 B.C.), in Historia, n. 1.2, Stuttgart, Steiner.

Günther 2016 - S. Günther, Sonderwirtschaftszonen. Antike Konzeptionen und Konstruktionen am Beispiel des athenischen Piräus, in K. Droß-Krupe - S. Föllinger - K. Ruffing (a cura di), Antike Wirtschaft und ibre kulturelle Prägung - The Cultural Shaping of the Ancient Economy, Wiesbaden, Harrassowitz.

Hansen 1982 - M.H. Hansen, The Athenian Ecclesia and the Assembly-Place on the Pnix, in GRBS, n. 23.3, (1982), 241-249.

Hansen-Nielsen 2004 - M.H. Hansen, T.H. Nielsen (a cura di), An Inventory of Archaic and Classical Poleis, Oxford - New York, Oxford University Press.

Hopper 1961 - R.J. Hopper, 'Plain', 'Shore', and 'Hill' in Early Athens, in ABSA, n. 56, Athens - London, British School at Athens.

Huxley 1973 - G. Huxley, Aristotle as Antiquary, in GRBS, n. 14.3, Durham (N.C.), Duke University, Department of Classics.

Kalimitzis 2000 - K. Kalimitzis, Aristotle on Political Enmity and Disease, Albany, State University of New York Press.

Kern 1900 - O. Kern, Die Inschriften von Magnesia am Maeander, Berlin, Reimer. 
Lintott $2013^{2}$ - A. Lintott, Violence, Civil Strife and Revolution in the Classical City, 750330 BC, London-Canberra, Croom Helm.

Lintott 2018 - A Lintott, Aristotle's Political Philosophy in its Historical Context: A New Translation and Commentary on Politics Books 5 and 6, London, Routledge.

Loddo 2016 - L. Loddo, Cambiamenti costituzionali nei Philippika di Teopompo di Chio, in IncidAntico, n. 14.2, Napoli, Luciano.

Loddo 2019 - L. Loddo, La dinamica isola-perea nelle strategie di rientro in patria di esuli e fuoriusciti greci fra $V$ e IV secolo a.C.: il caso di Clazomene, in M.E. Ruggerini - V. Szóke - M. Deriu (a cura di), Isole settentrionali, Isole mediterranee. Letteratura e società, Milano, Prometheus.

Low 2008 - P. Low, The Athenian Empire, Edinburgh, Edinburgh University Press.

Ma 2009 - J. Ma, Empire, Statuses and Realities, in J. Ma - N. Papazarkadas - R. Parker (a cura di), Interpreting the Athenian Empire, London, Duckworth 2009.

Mattingly 1996 - H.B. Mattingly, The Athenian Empire Restored: Epigraphic and Historical Studies, Ann Arbor, The University of Michigan Press.

Mazzarino 1947 - S. Mazzarino, Fra Oriente e Occidente. Ricerche di storia greca arcaica, Firenze, La Nuova Italia.

Meiggs-Lewis 1969 - R. Meiggs, D. Lewis (a cura di), A Selection of Greek Historical Inscriptions to the End of the Fifth Century B.C., Oxford, Clarendon Press.

Moggi 1976 - M. Moggi, I sinecismi interstatali greci: dalle origini al 338 a.C., Pisa, Edizione Marlin.

Morison 2016 - W.S. Morison, Theopompos of Chios (115), in Brill's New Jacoby, General Editor: Ian Worthington (Macquarie University). Consulted online on 25 March 2021: <url: http://0-dx.doi.org.opac.unicatt.it/10.1163/18735363_bnj_a115 >

Moysey 1981 - R.A. Moysey, The Thirty and the Pnyx, in AJA, n. 85.1, Boston, Boston University, Archaeological Institute of America.

Newman 1902 - W.L. Newman, The Politics of Aristotle, with an Introduction, Two Prefatory Essays and Notes Critical and Explanatory, Volume IV. Essay on Constitutions, Books VI-VII - Text and Notes, Oxford, Clarendon Press.

Papazarkadas 2009 - N. Papazarkadas, Epigraphy and the Athenian Empire: Reshuffling the Chronological Cards, in J. Ma, N. Papazarkadas, R. Parker (a cura di), Interpreting the Athenian Empire, London, Duckworth.

Parke 1985 - H.W. Parke, The Oracles of Apollo in Asia Minor, London, Croom Helm.

Pellegrin 2017 - P. Pellegrin, L'Excellence menacée. Sur la philosophie politique d'Aristote, Paris, Classiques Garnier.

Pezzullo 2012 - A. Pezzullo, La tryphe nella riflessione politica aristotelica, in M. Polito - C. Talamo (a cura di), Istituzioni e costituzioni in Aristotele tra storiografia e pensiero politico (Atti della Giornata Internazionale di Studio, Fisciano 30 settembre - 1 ottobre 2011), Roma, Tored.

Phillips Simpson 1998 - P.L. Phillips Simpson, A Philosophical Commentary on the Politics of Aristotle, Chapel Hill, University of North Carolina Press.

Piérart 1984 - M. Piérart, Deux notes sur la politique d'Athènes en mer Égée (428-425), in $B C H$, n. 108.1, Paris, De Boccard. 
Poddighe 2020 - E. Poddighe, "Like Men Driven from a Captured City" (Plut. Phoc. 28.4): Reconsidering the Displacement of the Disenfranchised Athenians to Thrace in 322 BC, in Pallas, n. 112, Toulouse, Presses Universitaires du Mirail.

Ragone 1996 - G. Ragone, La Ionia, l'Asia Minore, Cipro, in S. Settis (a cura di), I Greci. Storia, Cultura, Arte, Società. 2. Una storia greca. 1. Formazione, Torino, Einaudi. Ragone 2005 - G. Ragone, Colofone, Claro, Notio. Un contesto per Senofane, in M. Bugno (a cura di), Senofane ed Elea tra Ionia e Magna Grecia, Napoli, Luciano.

Rhodes 1981 - P.J. Rhodes, A Commentary on the Aristotelian Athenaion Politeia, Oxford, Clarendon Press.

Rhodes 2008 - P.J. Rhodes, After the Three-Bar Sigma Controversy: The History of Athenian Imperialism Reassessed, in CQ, n. 58.2, Oxford, Oxford University Press.

Robert 1962 - L. Robert, Villes d'Asie Mineure: études de géographie ancienne, Paris, De Boccard.

Rogan 2018 - E. Rogan, La Stásis dans la politique d'Aristote. La cité sous tension, Paris, Classiques Garnier.

Roy 1998 - J. Roy, The Threat from the Piraeus, in P. Cartledge - P. Millett - S. von Reden (a cura di), Kosmos. Essays in Order, Conflict and Community in Classical Athens, Cambridge, Cambridge University Press.

Rubinstein 2004 - L. Rubinstein, Ionia, in M.H. Hansen - T.H. Nielsen (a cura di), An Inventory of Archaic and Classical Poleis, Oxford - New York, Oxford University Press.

Ruzé 1997 - F. Ruzé, Délibération et pouvoir dans la cité grecque de Nestor à Socrate, Paris, Publications de la Sorbonne.

Schmidt-Hofner 2014 - S. Schmidt-Hofner, Politik räumlich denken. Herodots drei Parteien in Attika und das politische Imaginaire der Griechen, in HZ, n. 299.3, Munchen, Oldenbourg.

Schütrumpf-Gehrke 1996 - E. Schutrumpf, H.-J. Gehrke (a cura di), Aristoteles. Politik. Buch IV-VI, Berlin, Akademie Verlag.

Seibert 1979 - J. Seibert, Die politischen Flüchtlinge und Verbannten in der griechischen Geschichte: von den Anfangen bis zur Unterwerfung durch die Romer, Darmstadt, Wissenschaftliche Buchgesellschaft.

Shear 2011 - J. Shear, Polis and Revolution: Responding to Oligarchy in Classical Athens, Cambridge, Cambridge University Press.

Sordi 2006 - M. Sordi, Atene e l'unione fra Argo e Corinto, in C. Bearzot - F. Landucci (a cura di), Argo. Una democrazia diversa, Contributi di storia antica 4, Milano, Vita e Pensiero.

Talamo 1973 - C. Talamo, Per la storia di Colofone in età arcaica, in PP, n. 28, Napoli, Macchiaroli.

Tracy 2014 - S.V. Tracy, Down Dating Some Athenian Decrees with Three-Bar Sigma: A Palaeographic Approach, in ZPE, n. 190, Bonn, Habelt.

Von Reden 1995 - S. von Reden, The Piraeus - A World a Part, in GER, n. 42, Oxford, Clarendon Press.

Weed 2007 - R. Weed, Aristotle on Stasis: A Moral Psychology of Political Conflict, Berlin, Logos. 
Whitby 1984 - M. Whitby, The Union of Corinth and Argos: A Reconsideration, in Historia, n. 3, Stuttgart, Steiner.

Zatta 2010 - C. Zatta, Conflict, People, and City-Space: Some Exempla from Thucydides' History, in ClAnt, n. 30.2, Berkeley, University of California Press. 
